\title{
Multiphase-field modelling of concurrent grain growth and coarsening in complex multicomponent systems
}

\author{
P G Kubendran Amos ${ }^{\mathrm{a}, 1, *}$, Ramanathan Perumal ${ }^{\mathrm{a}, \mathrm{b}, 1}$, Michael Selzer ${ }^{\mathrm{a}, \mathrm{b}}$, Britta Nestler ${ }^{\mathrm{a}, \mathrm{b}}$ \\ ${ }^{a}$ Institute of Applied Materials (IAM-CMS), Karlsruhe Institute of Technology (KIT), \\ Strasse am Forum 7, 76131 Karlsruhe, Germany \\ ${ }^{b}$ Institute of Digital Materials Science (IDM), Karlsruhe University of Applied Sciences, \\ Moltkestr. 30, 76133 Karlsruhe, Germany
}

\begin{abstract}
Phase-field modelling of microstructural evolution in polycrystalline systems with phaseassociated grains has largely been confined to continuum-field models. In this study, a multiphasefield approach, with a provision for introducing grain boundary and interphase diffusion, is extended to analyse concurrent grain growth and coarsening in multicomponent polycrystalline microstructures with chemically-distinct grains. The effect of the number of phases and components on the kinetics of evolution is investigated by considering binary and ternary systems of duplex and triplex microstructures, along with a single phase system. It is realised that the mere increase in the number of phases minimises the rate of concurrent grain growth and coarsening. However, the effect of components is substantially dependent on the respective kinetic coefficients. This work unravels that the disparity in the influence of phases and components is primarily due to the corresponding change introduced in the transformation mechanism. While the raise in number of phases convolutes the diffusion paths, the increase in number of component effects the rate of evolution through the interdiffusion, which introduces interdependency in the diffusing chemical-species. Additionally, the role of phase-fractions on the transformation rate of triplex microstructure is studied, and correspondingly, the interplay of interfaceand diffusion-governed evolution is elucidated. A representative evolution of three-dimensional triplex microstructure with equal phase-fraction is comparatively analysed with the evolution of corresponding two-dimensional setup.
\end{abstract}

Keywords: Concurrent grain growth and coarsening, duplex and triplex microstructure, 
microstructural coarsening, multiphase grain growth, multiphase-field modelling

\section{Introduction}

A microstructure generally comprises of phases which are chemically and (/or) structurally different from one another. Often, in highly-applicable materials, the crystallographic orientation of these phases locally vary. Although a similar crystallographic relation prevails amongst the phases, the overall disparity in the orientation facilitates the cognisance of grains. In other words, a grain represents a section of a polycrystalline microstructure wherein the phases collectively exhibit an identical orientation. The difference in the directional alignment of the phases across the grains introduces grain boundaries. The energy density of a grain boundary is dictated by the degree of mis-orientation between the corresponding grains [1]. Since a definite energy is associated with every grain boundary, under suitable thermodynamic condition, a microstructure evolves to reduce the overall energy by minimising the boundary area. This evolution is commonly perceived as grain growth.

In a conventional polycrystalline material, each grain individually represents the entire microstructure by encompassing all the constituent phases in exact proportion. Therefore, despite the difference in the orientation, the grains are chemically identical. Under such condition, grain growth primarily involves interface migration, as the phase-fraction is independently preserved in each grains. This seemingly direct mechanism of grain growth gets convoluted when the grains are no longer chemically identical [2, 3]. Particularly, since a simple interface migration would disturb the characteristic phase-fraction of the microstructure.

\subsection{Multiphase polycrystalline materials}

In certain multiphase polycrystalline system, grains are chemically distinct, and are exclusively associated with one of the constituent phases [4, 5]. For instance, a duplex microstructure of phase- $\alpha$ and $-\beta$, wherein a grain belongs to either one of the two phases [6, 7]. These

\footnotetext{
*P G Kubendran Amos

Email address: prince.amos@kit .edu (P G Kubendran Amos)

${ }^{1}$ The authors contributed equally.
} 
phase-associated grains are increasingly reported to improve the applicability of a material by enhancing its properties. In ceramic composites, predominantly involving alumina, it is identified that the chemically distinct grains significantly improve the mechanical properties by increasing the crack resistance through localised bridging [8]. Furthermore, a desired combination of toughness, ductility and hardness is achieved in steels for automotive applications through the microstructure with grains exclusively associated with a constituent phase [9, 10]. Due to the increasing prominence of the multiphase polycrystalline materials in wide range of applications, these systems are extensively analysed to enhance the current understanding of its microstructural evolution.

In multiphase polycrystalline system, wherein the grains are exclusively associated with individual phases, a regular grain growth dictated by grain volume, number of neighbours, and other related factors, invariably results in a non-physical phase-change. Particularly, when a grain, favoured by its geometrical and topological features, grows at the expense of a neighbouring grain of different chemical feature (phase), the definite phase-fraction of the microstructures gets altered. Therefore, the seemingly direct mechanism of grain growth significantly changes to avert any deviation from the original phase-fraction [11]. In other words, while a grain of a given phase- $\alpha$ disappears in-keeping with the regular grain growth, the volume of an another preferred $\alpha$-grain correspondingly increases to ensure the overall volume-fraction of the phase remains conserved. This unique evolution, therefore, reflects grain growth and coarsening through the decrease in the number of grains, and unchanged phase-fraction, respectively. Accordingly, in addition to the interface migration, grain growth in the multiphase polycrystalline material is governed by the efficient mass transfer which ensures the time-invariant volume fractions of the constituent phases. In the present work, this concurrent grain growth and coarsening exhibited by the duplex and triplex microstructures of binary and ternary system are comparatively analysed.

\subsection{Phase-field modelling}

Since a microstructural evolution involves temporal change in the arrangement of the phases, an exhaustive investigation includes periodic observation of the evolving microstructure [12, 13]. 
Moreover, owing to the complex distribution of the phases, three-dimensional projections of the microstructures are employed to delineate the observed evolution [14, 15]. These conventional experimental analyses are extremely arduous, and expensive. Particularly, rather sluggish kinetics of the curvature-driven transformations, like grain growth and coarsening, compounds the difficulties of the experimental investigations. Therefore, theoretical treatments are often adopted as an efficient alternate for complementing and explicating the observed microstructural changes. Numerous techniques have been postulated to model microstructural changes [16, 17, 18]. Of these different techniques, phase-field approach is increasingly involved in modelling complex microstructural changes in both two- and three-dimensions [19, 20, 21].

A microstructural evolution is theoretically realised by the temporal change in the position of the interface. Therefore, modelling evolution of complex microstructure demands a highly sophisticated formulation, which substantially convolutes the technique. Phase-field approach averts such complications by shifting the focus from the position of the interface, and capturing the transformation through the spatio-temporal change in the newly introduced scalar variable called phase-field (or order parameter). By relaxing the need for tracking the interface, this tool adheres to a more elegant framework, which is conveniently extended to encompass different aspects of a given transformation [22, 23]. Due to its convincing formulation, and the ability to recover analytical (sharp-interface) solutions and physical laws, phase-field approach has widely been adopted to model microstructural changes including solidification [24, 25] and solid-state phase transformation [26, 27]. Furthermore, since this technique coherently captures the influences of curvature, grain growth [28, 29] and other morphological changes ensuing a microstructure in chemical equilibrium are analysed through the phase-field approach [30, 31]. Correspondingly, the phase-field models have been employed to examine microstructural evolution in multiphase polycrystalline systems [32, 33, 34]. Despite the extensive analyses in these works, the underpinning numerical treatments follow a definite framework, which deviate from technique adopted in present investigation. In order to elucidate the subtle differences in the current modelling approach, existing formulations are concisely discussed. 


\subsubsection{Continuum-field model}

Initial attempts to model microstructural evolution in multiphase polycrystalline system consider a two-phase setup, wherein the individual grains are distinguished through a definite order parameter [32, 35, 36, 37]. The two-phase polycrystalline system is described by the spatiallydiffering scalar variables $\left\{\left(\eta_{1}^{\alpha}(\boldsymbol{x}), \eta_{2}^{\alpha}(\boldsymbol{x}), \cdots, \eta_{q_{\alpha}}^{\alpha}(\boldsymbol{x})\right),\left(\eta_{1}^{\beta}(\boldsymbol{x}), \eta_{2}^{\beta}(\boldsymbol{x}), \cdots, \eta_{q_{\beta}}^{\beta}(\boldsymbol{x})\right), c(\boldsymbol{x})\right\}$. The superscript of the order parameter, $\eta_{i}^{\alpha}$ and $\eta_{j}^{\beta}$, represents the phases, while the subscript is used to distinguish the phases. A continuous variable representing the concentration, $c(\boldsymbol{x})$, is appended to impose the diffuse-governed evolution of the phases. Based on these scalar variables, and their corresponding spatial changes, the energy-density of the two-phase polycrystalline system of volume $V$ is expressed as a functional,

$$
\begin{aligned}
F\left(\eta_{i}^{\alpha}, \eta_{i}^{\beta}, \boldsymbol{\nabla} \eta_{i}^{\alpha}, \boldsymbol{\nabla} \eta_{i}^{\beta}, c\right)=\int_{V} \mathrm{~d} V & {\left[f_{0}\left(\eta_{i}^{\alpha}, \eta_{i}^{\beta}, c\right)+k_{c}|\nabla c|^{2}+\sum_{i=1}^{q_{\alpha}} k_{i}^{\alpha}\left|\nabla \eta_{i}^{\alpha}\right|^{2}\right.} \\
& \left.+\sum_{i=1}^{q_{\beta}} k_{i}^{\beta}\left|\nabla \eta_{i}^{\beta}\right|^{2}\right] \quad \text { where } i \in\left\{1,2, \cdots, q_{\alpha, \beta}\right\} .
\end{aligned}
$$

This formulation includes gradient of the concentration, $\nabla c$, and phase-associated order parameters, $\nabla \eta_{i}^{\alpha}$ and $\nabla \eta_{i}^{\beta}$. The phenomenological constant are represented by $k_{c}, k_{i}^{\alpha}$ and $k_{i}^{\beta}$. First term of the right side of the functional in Eqn. (1) is written as

$$
\begin{aligned}
f_{0}\left(\eta_{i}^{\alpha}, \eta_{i}^{\beta}, c\right) & =f_{\mathrm{ch}}\left(c, c_{\mathrm{eq}}^{\alpha}, c_{\mathrm{eq}}^{\beta}\right)+f_{\alpha}\left(c, c_{\mathrm{eq}}^{\beta}, \eta_{i}^{\alpha}\right) \\
& +f_{\beta}\left(c, c_{\mathrm{eq}}^{\alpha}, \eta_{i}^{\beta}\right)+f_{\alpha \beta}\left(\eta_{i}^{\alpha}, \eta_{i}^{\beta}\right),
\end{aligned}
$$

where $f_{\mathrm{ch}}\left(c, c_{\mathrm{eq}}^{\alpha}, c_{\mathrm{eq}}^{\beta}\right)$ is the energy contribution based on the concentration which is written as a Landau polynomial, and includes the equilibrium compositions of phase- $\alpha$ and $-\beta, c_{\mathrm{eq}}^{\alpha}$ and $c_{\mathrm{eq}}^{\beta}$ ), respectively. The equilibrium concentrations are constants and vary with the chemical makeup of the system. While the terms $f_{\alpha}\left(c, c_{\mathrm{eq}}^{\beta}, \eta_{i}^{\alpha}\right)$ and $f_{\beta}\left(c, c_{\mathrm{eq}}^{\alpha}, \eta_{i}^{\beta}\right)$ couple the order parameters with the continuous concentration, $f_{\alpha \beta}\left(\eta_{i}^{\alpha}, \eta_{i}^{\beta}\right)$ in Eqn. (2) accounts for the order parameters in the interface separating the grain- $i$ of phase- $\alpha$ and $-\beta$.

Subsequent work extends the non-gradient term $f_{0}\left(\eta_{i}^{\alpha}, \eta_{i}^{\beta}, c\right)$ in Eqn. (1) as

$$
\begin{aligned}
f_{0}\left(\eta_{i}^{\alpha}, \eta_{j}^{\alpha}, \eta_{i}^{\beta}, \eta_{j}^{\beta}, c\right) & =f_{\mathrm{ch}}\left(c, c_{\mathrm{eq}}^{\alpha}, c_{\mathrm{eq}}^{\beta}\right)+f_{\alpha}\left(c, c_{\mathrm{eq}}^{\alpha}, c_{\mathrm{eq}}^{\beta}, \eta_{i}^{\alpha}\right) \\
& +f_{\beta}\left(c, c_{\mathrm{eq}}^{\alpha}, c_{\mathrm{eq}}^{\beta}, \eta_{i}^{\beta}\right)+f_{\alpha \alpha}\left(\eta_{i}^{\alpha}, \eta_{j}^{\alpha}\right)+f_{\beta \beta}\left(\eta_{i}^{\beta}, \eta_{j}^{\beta}\right)+f_{\alpha \beta}\left(\eta_{i}^{\alpha}, \eta_{i}^{\beta}\right),
\end{aligned}
$$


and include equilibrium composition of the both the phases in the terms coupling the order parameter with the concentration [33]. Furthermore, interfaces between the grains of the identical phases are explicitly formulated through $f_{\alpha \alpha}\left(\eta_{i}^{\alpha}, \eta_{j}^{\alpha}\right)$ and $f_{\beta \beta}\left(\eta_{i}^{\beta}, \eta_{j}^{\beta}\right)$, along with the existing treatment of the chemically dissimilar grains. This extended formulation of $f_{0}\left(\eta_{i}^{\alpha}, \eta_{i}^{\beta}, c\right)$ obviates the need for the inclusion of concentration gradient in the functional, and consequently, the overall energy density of the system is expressed as

$$
F\left(\eta_{i}^{\alpha}, \eta_{i}^{\beta}, \boldsymbol{\nabla} \eta_{i}^{\alpha}, \boldsymbol{\nabla} \eta_{i}^{\beta}, c\right)=\int_{V} \mathrm{~d} V\left[f_{0}\left(\eta_{i}^{\alpha}, \eta_{i}^{\beta}, c\right)+\sum_{i=1}^{q_{\alpha}} k_{i}^{\alpha}\left|\nabla \eta_{i}^{\alpha}\right|^{2}+\sum_{i=1}^{q_{\beta}} k_{i}^{\beta}\left|\nabla \eta_{i}^{\beta}\right|^{2}\right]
$$

Despite the efficient formulation of the energy functional, it is vital to realise that the continuous concentration is directly coupled to the order parameter in both Eqns. (2) and (3).

Recent works report on numerical treatment of systems with more than two phases and one independent concentration [34, 38, 39]. The extended energy-functional, which encompasses a system $N$ phases and $k$ chemical species, is written as

$$
\begin{aligned}
F\left(\eta_{i}^{\alpha}, \eta_{i}^{\beta}, \cdots, \eta_{i}^{N}, \nabla \eta_{i}^{\alpha}, \nabla \eta_{i}^{\beta}, \cdots, \nabla \eta_{i}^{N}, \boldsymbol{c}\right) & =\int_{V} \mathrm{~d} V\left[f_{\mathrm{ch}}\left(\eta_{i}^{\alpha}, \eta_{i}^{\beta}, \cdots, \eta_{i}^{N}, \boldsymbol{c}\right)\right. \\
& \left.+f_{\mathrm{pot}}\left(\eta_{i}^{\alpha}, \eta_{i}^{\beta}, \cdots, \eta_{i}^{N}\right)+f_{\mathrm{gr}}\left(\boldsymbol{\nabla} \eta_{i}^{\alpha}, \boldsymbol{\nabla} \eta_{i}^{\beta}, \cdots, \boldsymbol{\nabla} \eta_{i}^{N}\right)\right] .
\end{aligned}
$$

Despite the similarity in the interpretation of the scalar variables, as opposed to the aforementioned techniques, this approach introduces a definite interpolation function to define the concentrationbased contribution, $f_{\mathrm{ch}}\left(\eta_{i}^{\alpha}, \eta_{i}^{\beta}, \cdots, \eta_{i}^{N}, \boldsymbol{c}\right)$, and simultaneously couple the concentration with order parameter [40]. Particularly, the Landau polynomial based on the continuous concentration is replaced by an approximation of the free-energy density. This formulation of the concentrationbased contribution, in relation to the present treatment, will be discussed after delineating the adopted model.

Irrespective of the disparities in the description of the various energy contributions, the system is allowed to evolve towards a phenomenological decrease in the overall energy-density. Correspondingly, in all the aforementioned techniques, the temporal evolution of the order pa- 
rameter is expressed as

$$
\frac{\partial \eta_{i}^{\alpha}}{\partial t}=-\mathcal{L}\left[\frac{\delta F}{\delta \eta_{i}^{\alpha}}\right]
$$

where $\mathcal{L}$ governs the mobility of the evolving variable. Since the concentration fundamentally differs from the non-conserved order parameter, its evolution is reads

$$
\frac{\partial c_{i}}{\partial t}=\boldsymbol{\nabla} \cdot\left[\sum_{\alpha}^{N} \sum_{i=1}^{k-1} M_{i}^{\alpha} \nabla \frac{\delta F}{\delta c_{i}}\right] .
$$

The combined evolution of order parameter and concentration renders a microstructural transformation which includes the aspects of both grain growth and coarsening. In other words, while the evolution of order parameters in Eqn. (6) accounts for grain growth, the volume fractions of the evolving phases are preserved by the concentration.

All the techniques discussed in this section, treat the order parameter as an independent variable with limited physical attribution. Numerical approaches with such treatment of the order parameters are generally referred to as continuum-field models [41].

\subsection{Plausible multiphase-field approach}

Multiphase-field model, as opposed to continuum-field approach, consider its corresponding scalar variable, called phase-field, to represent local volume-fraction of a grain (phase) [42]. This consideration imposes a constraint that, in a given position, the sum of all phase-field should be equal to one. The interpretation of the associated scalar variables is the primary difference between the continuum-field and multiphase-field model [43]. The consequence of the physical attributes to phase-field will be elucidated once the model is introduced.

Phase-field techniques hitherto adopted to analyse microstructural evolution of multiphase polycrystalline systems have predominantly been confined continuum-field model. However, based on the existing works wherein multiphase-field approach has been adopted to address similar microstructural changes, a formulation to model microstructural changes in a system of $N$ phases can be conceived [44, 45, 46]. This possible multiphase-field treatment is briefly discussed in this section in order to explicate its difference from the present framework. 
A system of $N$ phases, in a multiphase-field approach, is defined by appropriate scalar variables, which are conventionally written as a tuple,

$$
\phi=\left\{\phi_{1}, \phi_{2}, \ldots, \phi_{N}\right\}
$$

Such representation is deemed permissible due to the interdependency between phase-fields imposed by the physical attribution. The $N$-tuple representation, as in Eqn. (8), is however in sufficient to describe a system wherein numerous grains are associated with each of the phases. Accordingly, the general consideration of phase-field should be extended as

$$
\phi=\{\underbrace{\left\{\phi_{\alpha}^{1}, \phi_{\alpha}^{2} \ldots \phi_{\alpha}^{q_{\alpha}}\right\}}_{\phi_{\alpha}}, \underbrace{\left\{\phi_{\beta}^{1}, \phi_{\beta}^{2} \ldots \phi_{\beta}^{q_{\beta}}\right\}}_{\phi_{\beta}} \ldots \underbrace{\left\{\phi_{N}^{1}, \phi_{N}^{2} \ldots \phi_{N}^{q_{N}}\right\}}_{\phi_{N}}\},
$$

where number of grains in each phase is denoted by $q_{\Theta}$ with $\Theta \in\{\alpha, \beta \cdots N\}$. The coarsening aspect of the microstructural transformation which ensures the volume-fraction of the phases are unchanged is incorporated by introducing an additional non-conserved scalar variable, $\rho^{\Theta}$. While $\phi_{\alpha}^{i}$ represents the volume fraction of grain- $i$ of phase- $\alpha$, the volume fraction of the entire phase in the microstructure is assumed to be accounted by $\rho^{\alpha}$. The newly introduced variable, akin to phase-field, is expressed as

$$
\boldsymbol{\rho}=\left\{\rho^{\alpha}, \rho^{\beta} \cdots \rho^{N}\right\}
$$

where $\rho^{\Theta}$ assumes a definite value in a phase irrespective of the differences in grains.

The overall energy functional of the system, in this multiphase-field framework, is expressed as

$$
F(\boldsymbol{\phi}, \boldsymbol{\rho}, \boldsymbol{\nabla} \boldsymbol{\phi}, \boldsymbol{\nabla} \boldsymbol{\rho})=\int_{V} \mathrm{~d} V\left[f_{0}(\boldsymbol{\phi}, \boldsymbol{\rho})+f_{\boldsymbol{\phi}}(\boldsymbol{\nabla} \boldsymbol{\phi})+f_{\boldsymbol{\rho}}(\boldsymbol{\nabla} \boldsymbol{\rho})\right]
$$

wherein along with phase-field, the gradient in the coarsening variable, $\rho$, is appended. Owing to the nature of these scalar variables, their corresponding temporal evolution is formulated as

$$
\tau \frac{\partial \phi_{\alpha}^{i}}{\partial t}=-\left[\frac{\delta F}{\delta \phi_{\alpha}^{i}}+\lambda\right]
$$

and

$$
\frac{\partial \rho^{\alpha}}{\partial t}=\nabla \cdot\left[M \nabla \frac{\delta F}{\delta \rho^{\alpha}}\right]
$$


where $\tau$ and $\lambda$ correspond to the relaxation parameter and Lagrange multiplier, which ensures that $\sum_{\alpha} \sum_{i} \phi_{\alpha}^{i}=1$ at any location in the system. Moreover, $M$ dictates the mobility of the conserved variable. The combination of the evolution, in accordance to Eqn. (12) ad (13) render a unique grain growth wherein the volume-fraction of the phases are preserved all-through the

Adopting the description of phase-field in Eqn. (9), and following the existing works, which were adopted for modelling phase transformations and two-phase curvature-driven evolutions, the overall energy density of a $k$-component system with $N$-phase dissociated into $\bar{N}\left(=\sum_{\alpha}^{N} q_{\alpha}\right)$ grains is written as a combination of bulk and interface contribution [47, 26, 48]. Correspondingly, energy-density functional of a system of volume $V$ is expressed as

$$
\begin{aligned}
\mathcal{F}(\boldsymbol{\phi}, \boldsymbol{\nabla} \boldsymbol{\phi}, \boldsymbol{c}) & =\mathcal{F}_{\text {int }}(\boldsymbol{\phi}, \boldsymbol{\nabla} \boldsymbol{\phi})+\mathcal{F}_{\text {bulk }}(\boldsymbol{\phi}, \boldsymbol{c}) \\
& =\int_{V} f_{\text {int }}(\boldsymbol{\phi}, \boldsymbol{\nabla} \boldsymbol{\phi})+f_{\text {bulk }}(\boldsymbol{\phi}, \boldsymbol{c}) \mathrm{d} V,
\end{aligned}
$$

where $\mathcal{F}_{\text {bulk }}(\phi, c)$ and $\mathcal{F}_{\text {int }}(\phi, \nabla \phi)$ respectively denote the contributions of the bulk region, and interface separating the different phase-associated grains.

\subsection{Interface contribution}

The contribution of the diffuse region separating the phase-associated grains is written as

$$
f_{\text {int }}(\phi, \nabla \phi)=\varepsilon a(\phi, \nabla \phi)+\frac{1}{\varepsilon} w(\phi)
$$

where $\varepsilon a(\phi, \nabla \phi)$ and $\frac{1}{\varepsilon} w(\phi)$ represent the gradient-energy term and the penalising obstacletype potential [47]. In Eqn. (15), $\varepsilon$ is a length-scale parameter which dictates the width of the diffuse interface. 
The gradient-energy contribution, in the present multiphase model, is written as the summation of all possible pairwise interaction between the grains with similar and dissimilar phase association [49]. Accordingly, the gradient-energy density, for the complex multiphase microstructure is formulated as

$$
\varepsilon a(\boldsymbol{\phi}, \boldsymbol{\nabla} \boldsymbol{\phi})=\sum_{\alpha \leq \beta}^{N} \sum_{m \leq n}^{q_{\alpha}} \gamma_{\alpha \beta}^{m n}\left|\mathcal{Q}_{\alpha \beta}^{m n}\right|^{2},
$$

where $\gamma_{\alpha \beta}^{m n}$ is the interfacial energy of the grain boundary separating grains $m$ and $n$ of $\alpha-$ and $\beta$-phase, respectively. The gradient vector $\mathcal{Q}_{\alpha \beta}^{m n}$, in above Eqn. (16), is ascertained by

$$
\mathcal{Q}_{\alpha \beta}^{m n}=\phi_{\alpha}^{m} \nabla \phi_{\beta}^{n}-\phi_{\beta}^{n} \nabla \phi_{\alpha}^{m}
$$

The gradient-energy formulation in Eqn. (16) is restricted to the isotropic condition. However, by augmenting a pre-factor, $\bar{c}\left(\mathcal{Q}_{\alpha \beta}^{m n}\right)$, which is a function of the gradient vector, anisotropy in the interfacial energy can be introduced [50].

In the current multiphase-field technique, the penalising potential is efficiently adopted by imposing the constraint through the Gibbs simplex. The penalising criterion in the Gibbs simplex is expressed as

$$
\mathcal{G}=\left\{\phi \in \mathbb{R}^{\bar{N}}: \sum_{\alpha}^{N} \sum_{m}^{q_{\alpha}} \phi_{\alpha}^{m}=1, \phi_{\alpha}^{m} \geq 0\right\},
$$

where $\bar{N}$ is the total number of phase-associated grains in the system. The constraint $\sum_{\alpha}^{N} \sum_{m}^{q_{\alpha}} \phi_{\alpha}^{m}=$ 1 renders the primary distinction between the multiphase-field and continuum field treatments [43]. By imposing this condition through the Gibbs simplex, the obstacle-type potential is written as

$$
\frac{1}{\varepsilon} \omega(\phi)= \begin{cases}\frac{1}{\varepsilon}\left(\frac{16}{\pi^{2}} \sum_{\alpha \leq \beta} \sum_{m \leq n} \gamma_{\alpha \beta}^{m n} \phi_{\alpha}^{m} \phi_{\beta}^{n}+\sum_{\alpha \leq \beta \leq \delta} \sum_{m \leq n \leq p} \gamma_{\alpha \beta \delta}^{m n p} \phi_{\alpha}^{m} \phi_{\beta}^{n} \phi_{\delta}^{p}\right), & \phi \in \mathcal{G} \\ \infty & \phi \notin \mathcal{G} .\end{cases}
$$

While the first term in the obstacle-type potential includes all interfaces, the second higherorder terms prevent the formation of the spurious third-phases. Moreover, by introducing the constraint

$$
\gamma_{\alpha \alpha}^{m m}=\gamma_{\alpha \alpha \alpha}^{m m m}=0 \quad \forall\left\{\alpha \in[N]: m \in\left[q_{\alpha}\right]\right\} .
$$

in Eqns. (16) and (19), any non-physical contributions is dismissed. 


\subsection{Volume preservation by bulk contribution}

During the concurrent grain growth and coarsening of complex multiphase microstructure, the volume fractions of the phases are preserved. However, analogous to the regular grain growth, number of grains associated with each phase decreases with time. In other words, in a multiphase-field framework, assuming that the evolution is devoid of any phase-change, the individual phase-fields $\left(\phi_{\alpha}^{m}\right)$ are non-conserved, while $\phi_{\alpha}$ which represents the overall volumefraction of phase $-\alpha$ is conserved all through the transformation. The volume of the phases, in the current technique, is preserved by introducing a bulk energy-density contribution [19, 30]. The total contribution of the grains constituting the multiphase microstructure is expressed as

$$
f(\boldsymbol{\phi}, \boldsymbol{c})=\sum_{\alpha}^{N} \sum_{m}^{q_{\alpha}} f_{m}^{\alpha}\left(\boldsymbol{c}_{m}^{\alpha}\right) h\left(\phi_{\alpha}^{m}\right),
$$

where $f_{m}^{\alpha}\left(\boldsymbol{c}_{m}^{\alpha}\right)$ is the free-energy density of an individual $\alpha$-grain. These individual contributions are interpolated using the function $h\left(\phi_{\alpha}^{m}\right)$, which satisfies the condition $h(0)=0$, $h(1)=1$ and $\partial h\left(\phi_{\alpha}^{m}\right) /\left(\partial \phi_{\alpha}^{m}\right)=0$ in $\left\{\phi_{\alpha}^{m} \notin(0,1)\right\}$. Therefore, interpolation function of the form $h\left(\phi_{\alpha}^{m}\right)=\frac{\sum_{m}^{q_{\alpha}}\left|\phi_{\alpha}^{m}\right|^{2}}{\sum_{\alpha}^{N} \sum_{m}^{q_{\alpha}}\left|\phi_{\alpha}^{m}\right|^{2}}$ is adopted [40].

The free-energy density of individual grains are described based on its corresponding concentration. Owing to the multicomponent nature of the system, the concentration, akin to phasefield, is expressed as a tuple vector

$$
\boldsymbol{c}_{m}^{\alpha}=\left\{c_{m: i}^{\alpha}, c_{m: j}^{\alpha}, \ldots, c_{m: k}^{\alpha}\right\}
$$

with $k$ representing the number of chemical species including matrix (solvent) and $c_{m: i}^{\alpha}$, the molefraction of component $i$ in grain $m$ of phase $-\alpha$. Since the grains of a given phase, have identical chemical make-up and, are distinguished only by the respective non-conserved scalar variable, the concentration can be generalised as

$$
\boldsymbol{c}_{1}^{\alpha}=\cdots=\boldsymbol{c}_{m}^{\alpha}=\cdots=\boldsymbol{c}_{q_{\alpha}}^{\alpha} \equiv \boldsymbol{c}^{\alpha}=\left\{c_{i}^{\alpha}, c_{j}^{\alpha}, \ldots, c_{k}^{\alpha}\right\}
$$

Accordingly, the free-energy contribution of the bulk phases now reads

$$
f(\boldsymbol{\phi}, \boldsymbol{c})=\sum_{\alpha}^{N} \sum_{m}^{q_{\alpha}} f_{m}^{\alpha}\left(\boldsymbol{c}^{\alpha}\right) h\left(\phi_{\alpha}^{m}\right),
$$


wherein the concentration is solely phase-dependent. Conventionally, the free-energy densities of the individual phase-associated grains are influenced by temperature as well. Since this analysis predominantly focuses on isothermal transformations, the effect of temperature on freeenergy density is not included in Eqn. (24).

In the diffuse region separating the bulk phases, it is assumed that the grains, with spatially varying volume-fractions, co-exist and are in chemical equilibrium with each other [51, 52]. This equilibrium is characterised by the equivalence in the chemical potential. Therefore, the local equilibrium between the grains of same and different phases is written as

$$
\begin{gathered}
\frac{\partial f_{m}^{\alpha}}{\partial c_{i}^{\alpha}}=\frac{\partial f_{n}^{\alpha}}{\partial c_{i}^{\alpha}}=\cdots=\frac{\partial f_{m}^{\beta}}{\partial c_{i}^{\beta}}=\frac{\partial f_{n}^{\beta}}{\partial c_{i}^{\beta}}=\cdots=\frac{\partial f_{m}^{N}}{\partial c_{i}^{N}}=\frac{\partial f_{n}^{N}}{\partial c_{i}^{N}} \\
\mu_{i}^{\alpha}=\mu_{i}^{\beta}=\cdots=\mu_{i}^{N}=\mu_{i},
\end{gathered}
$$

where $\mu_{i}$ represent the chemical potential of component $i$. Owing to its local-equivalence, the chemical potential is treated as the continuous variable across the interface. Moreover, based on the equilibrated condition in Eqn. (25), the concentration is interpolated as

$$
\boldsymbol{c}=\sum_{\alpha}^{N} \sum_{m}^{q_{\alpha}} \boldsymbol{c}^{\alpha} h\left(\phi_{\alpha}^{m}\right),
$$

wherein, due to the identical interpolation function, the concentration varies smoothly across the interface similar to the free-energy density of the individual grains.

\subsection{Phase-field evolution}

As opposed to the conventional theoretical technique, the spatio-temporal evolution of the phase-field translates into the microstructural transformation in the phase-field approach. This obviates the need for the interface-tracking and thereby, significantly reduces the ensuing complexity. The evolution of the phase-field is dictated by the phenomenological decrease in the overall energy-density of the system. Accordingly, for the current formulation, the evolution of the phase-field is expressed as

$$
\begin{aligned}
\tau \varepsilon \frac{\partial \phi_{\alpha}^{m}}{\partial t} & =-\frac{\partial \mathcal{F}(\boldsymbol{\phi}, \boldsymbol{\nabla} \boldsymbol{\phi}, \boldsymbol{c})}{\partial \phi_{\alpha}^{m}} \\
& =\varepsilon\left[\boldsymbol{\nabla} \cdot \frac{\partial a(\boldsymbol{\phi}, \boldsymbol{\nabla} \boldsymbol{\phi})}{\partial \boldsymbol{\nabla} \phi_{\alpha}^{m}}-\frac{\partial a(\boldsymbol{\phi}, \boldsymbol{\nabla} \boldsymbol{\phi})}{\partial \phi_{\alpha}^{m}}\right]-\frac{1}{\varepsilon}\left[\frac{\partial w(\boldsymbol{\phi})}{\partial \phi_{\alpha}^{m}}\right]-\left[\frac{f_{m}^{\alpha}\left(\boldsymbol{c}^{\alpha}, \phi_{\alpha}^{m}\right)}{\partial \phi_{\alpha}^{m}}\right]-\Lambda,
\end{aligned}
$$


where $\Lambda$ is the Lagrange multiplier which ensures $\sum_{\alpha} \phi_{\alpha}(\boldsymbol{x} \mid \boldsymbol{x} \in[V])=1$. The Lagrange multiplier is expressed as

$$
\Lambda=\frac{1}{\bar{N}} \sum_{\beta}^{N} \sum_{m}^{q_{\alpha}} \varepsilon\left[\boldsymbol{\nabla} \cdot \frac{\partial a(\boldsymbol{\phi}, \boldsymbol{\nabla} \boldsymbol{\phi})}{\partial \boldsymbol{\nabla} \phi_{\beta}^{m}}-\frac{\partial a(\boldsymbol{\phi}, \boldsymbol{\nabla} \boldsymbol{\phi})}{\partial \phi_{\beta}^{m}}\right]-\frac{1}{\varepsilon}\left[\frac{\partial w(\boldsymbol{\phi})}{\partial \phi_{\beta}^{m}}\right]-\left[\frac{f_{m}^{\beta}\left(\boldsymbol{c}^{\beta}, \phi_{\beta}^{m}\right)}{\partial \phi_{\beta}^{m}}\right] .
$$

In Eqn. (27), $\tau$ is the relaxation constant which sustains the stability of the interface during the temporal evolution [53].

\subsection{Thermodynamic driving-force}

The driving force which governs the temporal evolution of the phase-field, as formulated in Eqn. (27), is written as

$$
\begin{aligned}
\Delta f_{\alpha}^{m}(\boldsymbol{c}, \boldsymbol{\phi})= & \left\{\left[f_{m}^{\alpha}\left(\boldsymbol{c}^{\alpha}\right)-\sum_{i}^{k-1} \mu_{i}\left(\boldsymbol{c}^{\alpha}\right) c_{i}^{\alpha}\right] \frac{\partial h\left(\phi_{\alpha}^{m}\right)}{\partial \phi_{\alpha}^{m}}\right\} \\
& -\left(\frac{1}{\bar{N}-q_{\alpha}}\right)\left\{\sum_{\beta}^{N} \sum_{m}^{q_{\beta}}\left[f_{m}^{\beta}\left(\boldsymbol{c}^{\beta}\right)-\sum_{i}^{k-1} \mu_{i}\left(\boldsymbol{c}^{\beta}\right) c_{i}^{\beta}\right] \frac{\partial h\left(\phi_{\beta}^{m}\right)}{\partial \phi_{\beta}^{m}}\right\} \\
& -\left(\frac{1}{q_{\alpha}}\right)\left\{\sum_{n}^{q_{\alpha}}\left[f_{n}^{\alpha}\left(\boldsymbol{c}^{\alpha}\right)-\sum_{i}^{k-1} \mu_{i}\left(\boldsymbol{c}^{\alpha}\right) c_{i}^{\alpha}\right] \frac{\partial h\left(\phi_{\alpha}^{n}\right)}{\partial \phi_{\alpha}^{n}}\right\} .
\end{aligned}
$$

While the contribution of the chemically-dissimilar grains to the driving force is included in the second term on the right-hand side of Eqn. (29), the interactions between the grains of the same phase is appended as the third term. Interestingly, the contribution of an individual grain to the driving force can be viewed as the Legendre transform of the free-energy density. Therefore, by treating the driving force as the difference in the grand chemical-potential,

$$
f_{m}^{\alpha}\left(\boldsymbol{c}^{\alpha}\right)-\sum_{i}^{k-1} \mu_{i}\left(\boldsymbol{c}^{\alpha}\right) c_{i}^{\alpha} \equiv \psi_{\alpha}^{m}\left(\boldsymbol{c}^{\alpha}\right)
$$

phase-field models have been formulated [54, 48, 55].

Without losing the generality, the free-energy contribution of an individual grain, using the appropriate thermodynamic conjugate-pairs [56], can be expressed as

$$
f_{m}^{\alpha}\left(c^{\alpha}\right)=\sum_{i}^{k} \mu_{m: i}^{\alpha} c_{m: i}^{\alpha}
$$


Since $c_{m: i}^{\alpha}$ denotes the mole-fraction of component $i$ in the individual grain $m$ of phase $-\alpha$, the condition $\sum_{i}^{k} c_{m: i}^{\alpha}=1$ is fulfilled. By introducing this condition, the free-energy density in Eqn. (31) is written as

$$
f_{m}^{\alpha}\left(\boldsymbol{c}^{\alpha}\right)=\mu_{m: o}^{\alpha}+\sum_{i}^{k-1} \mu_{m: i}^{\tilde{\alpha}} c_{i}^{\alpha}
$$

where $\mu_{o}^{\alpha}$ is the chemical potential of the matrix component. Moreover, in Eqn. $32, \tilde{\mu}_{i}^{\alpha}=\mu_{m: i}^{\alpha}-$ $\mu_{m: o}^{\alpha}$ represents the diffusion potential. The driving-force contribution of the individual grain can be written based on this free-energy formulation and adopting the appropriate Legendre transform. Correspondingly, in the present framework, the contribution of a grain to the phasefield evolution is expressed

$$
\psi_{\alpha}^{m}\left(\boldsymbol{c}^{\alpha}\right)=\mu_{o}\left(\boldsymbol{c}^{\alpha}\right)+\underbrace{\sum_{i}^{k-1} \tilde{\mu}_{i}\left(\boldsymbol{c}^{\alpha}\right) c_{i}^{\alpha}-\sum_{i}^{k-1} \tilde{\mu}_{i}\left(\boldsymbol{c}^{\alpha}\right) c_{i}^{\alpha}}_{:=0} .
$$

The overall driving-force in Eqn. (29) now reads

$$
\begin{aligned}
\Delta f_{\alpha}^{m}(\boldsymbol{c}, \boldsymbol{\phi})=\left[\mu_{o}\left(\boldsymbol{c}^{\alpha}\right) \frac{\partial h\left(\phi_{\alpha}^{m}\right)}{\partial \phi_{\alpha}^{m}}\right] & -\left(\frac{1}{\bar{N}-q_{\alpha}}\right)\left\{\sum_{\beta}^{N} \sum_{m}^{q_{\beta}}\left[\mu_{o}\left(\boldsymbol{c}^{\beta}\right) \frac{\partial h\left(\phi_{\beta}^{m}\right)}{\partial \phi_{\beta}^{m}}\right]\right\} \\
& -\left(\frac{1}{q_{\alpha}}\right)\left\{\sum_{n}^{q_{\alpha}}\left[\mu_{o}\left(\boldsymbol{c}^{\alpha}\right) \frac{\partial h\left(\phi_{\alpha}^{n}\right)}{\partial \phi_{\alpha}^{n}}\right]\right\} .
\end{aligned}
$$

According to the above formulation, the difference in the matrix chemical-potential, which is dictated by the respective concentration, governs the evolution of the phase-field. However, during the concurrent grain growth and coarsening, the transformations are solely directed by the curvature $(\boldsymbol{K})$. Moreover, owing to the equilibrated condition in the diffuse interface, Eqn. 25, the deviation introduced by the curvature to the equilibrium chemical-potential is identical for all components [51, 52]. This equivalent change in the chemical potential, due to the curvature, is written as

$$
\mu_{o}(\boldsymbol{K}) \equiv \mu_{i}(\boldsymbol{K}) \quad \forall i
$$

Therefore, the curvature-dependent driving force which dictates the grain growth and Ostwald 
ripening in a complex microstructure is expressed as

$$
\begin{aligned}
\Delta f_{\alpha}^{m}(\boldsymbol{c}, \phi)=\left[\mu_{i}(\boldsymbol{K}) \frac{\partial h\left(\phi_{\alpha}^{m}\right)}{\partial \phi_{\alpha}^{m}}\right] & -\left(\frac{1}{\bar{N}-q_{\alpha}}\right)\left\{\sum_{\beta}^{N} \sum_{m}^{q_{\beta}}\left[\mu_{i}(\boldsymbol{K}) \frac{\partial h\left(\phi_{\beta}^{m}\right)}{\partial \phi_{\beta}^{m}}\right]\right\} \\
& -\left(\frac{1}{q_{\alpha}}\right)\left\{\sum_{n}^{q_{\alpha}}\left[\mu_{i}(\boldsymbol{K}) \frac{\partial h\left(\phi_{\alpha}^{n}\right)}{\partial \phi_{\alpha}^{n}}\right]\right\} .
\end{aligned}
$$

Analogous to the sharp-interface solutions, the microstructural evolution is governed, as indicated in the above Eqn. (36), by the disparity in the chemical potential introduced by the curvature.

\subsection{Evolution of governing chemical-potential}

The temporal change in the curvature-dependent chemical potential, which correspondingly drives the microstructural transformation, is formulated by considering the evolution of the respective concentration. The temporal evolution of a component- $i$, based on its influencingfactors, is expressed as

$$
\frac{\partial c_{i}\left(\mu_{i}(\boldsymbol{K}), \boldsymbol{\phi}\right)}{\partial t}=\left(\frac{\partial c_{i}}{\partial \mu_{i}(\boldsymbol{K})}\right)_{\phi_{\alpha}^{m}} \frac{\partial \mu_{i}(\boldsymbol{K})}{\partial t}+\left(\frac{\partial c_{i}}{\partial \phi_{\alpha}}\right)_{\mu_{i}} \frac{\partial \phi_{\alpha}^{m}}{\partial t}
$$

However, the evolution of the component $-i$ can be independently described depending on its flux which is induced by the influence of curvature on the chemical potential. In other words, curvature introduces a disparity in the equilibrium chemical-potential, which consequently actuates an atomic migration from the region of high potential to the low-potential sink. The concentration evolution can be formulated based on this flux. However, owing to the complexity of the microstructure, certain reasonable assumptions, largely pertaining to the diffusion coefficients, are made before formulating the concentration evolution.

Since the composition of the grains of a given phase is identical, the bulk diffusivity within the chemically-similar grains are considered equal,

$$
D_{\alpha: i}^{m}=D_{\alpha: i}^{n}=\cdots=D_{\alpha: i}^{q_{\alpha}}=D_{\alpha: i} \quad \forall\{\alpha \in[N]\}
$$

where $D_{\alpha: i}^{m}$ is the diffusion coefficient of component $-i$ in grain $m$ of phase- $\alpha$. In addition to the volume or bulk diffusion, the model is formulated to include grain boundary diffusion. It is 
assumed that the diffusion coefficients of the fluxes along the interface separating the grains of a given phase are equal. This assumption is expressed as

$$
D_{\alpha \alpha: i}^{m n}=D_{\alpha \alpha: i}^{m p}=\cdots=D_{\alpha \alpha: i}^{n p}=\cdots=D_{\alpha \alpha: i}^{q p}=D_{\alpha \alpha: i} \quad \forall\{n \in[q]: \alpha \in[N]\},
$$

where $D_{\alpha \alpha: i}^{m n}$ represents the diffusivity of component $-i$ along the interface between the grains $m$ and $n$ of phase $-\alpha$. In Eqn. (39) alone, grains $m, n, p$ and $q$ all pertain to phase- $\alpha$.

The concentration evolution of component- $i$, based on the atomic flux induced by the curvature, is expressed as

$$
\begin{aligned}
& \frac{\partial c_{i}\left(\mu_{i}(K)\right.}{\partial t}=\boldsymbol{\nabla} \cdot \sum_{j=1}^{k-1}\left[\sum_{\alpha=1}^{N} D_{\alpha: i}\left(\frac{\partial c_{i}^{\alpha}}{\partial \mu_{j}}\right) \sum_{m}^{q} h\left(\phi_{\alpha}^{m}\right) \nabla \mu_{j}(K)\right] \\
& +\nabla \cdot \sum_{j=1}^{k-1}\left\{\left(\sum_{\alpha}^{N} \boldsymbol{D}_{\alpha \alpha: i} \sum_{m<n}^{q, q}\left[\left(\frac{\partial c_{i}^{\alpha}}{\partial \mu_{j}}\right) h\left(\phi_{\alpha}^{m}\right)+\left(\frac{\partial c_{i}^{\beta}}{\partial \mu_{j}}\right) h\left(\phi_{\alpha}^{n}\right)\right] \phi_{\alpha}^{m} \phi_{\alpha}^{n}\right) \nabla \mu_{j}(K)\right\} \\
& +\nabla \cdot \sum_{j=1}^{k-1}\left\{\left(\sum_{\alpha}^{N} \sum_{\alpha<\beta}^{N} \boldsymbol{D}_{\alpha \beta: i} \sum_{m \leq n}^{q, q}\left[\left(\frac{\partial c_{i}^{\alpha}}{\partial \mu_{j}}\right) h\left(\phi_{\alpha}^{m}\right)+\left(\frac{\partial c_{i}^{\beta}}{\partial \mu_{j}}\right) h\left(\phi_{\beta}^{n}\right)\right] \phi_{\alpha}^{m} \phi_{\beta}^{n}\right) \nabla \mu_{j}(K)\right\},
\end{aligned}
$$

where the second and third terms on the right-hand side of the Eqn. (40) correspond to the migration along the boundaries of the grains of similar and the dissimilar phases. The interface diffusivities which govern the atomic fluxes along the grain boundaries are incorporated as a $N \times N$ symmetric matrix, which reads

$$
\boldsymbol{D}_{\alpha \beta: i}=\left[\begin{array}{cccc}
D_{\alpha \alpha: i} & D_{\alpha \beta: i} & \cdots & D_{\alpha N i} \\
& D_{\beta \beta: i} & \cdots & D_{\beta N: i} \\
& & \ddots & \vdots \\
& \text { sym. } & & D_{N N: i}
\end{array}\right] .
$$

This representation is enabled by the assumption in Eqn. (39). In other words, the interface diffusivity along the boundaries of the chemically-identical grains are represented by the diagonal entities of the matrix $\boldsymbol{D}_{\alpha \alpha: i}$, while the non-diagonal elements include diffusion coefficients along all the other grain boundaries. The proportionality constant, $\partial c_{i}^{\alpha} / \partial \mu_{j}$, which is the inverse of the second-derivative of the individual free-energy density, relates the diffusivity to the mobility of the component $-i$ in response to the chemical-potential gradient. 
By substituting Eqn. (40) in Eqn. (37), and re-arranging the terms, the temporal evolution of the curvature-dependent chemical potential which drives the phase-field evolution is written as

$$
\frac{\partial \mu_{i}(K)}{\partial t}=\left\{\boldsymbol{\nabla} \cdot\left[\sum_{j=1}^{k-1} \boldsymbol{M}(\phi) \nabla \mu_{j}(K)\right]-\sum_{\alpha}^{N} \sum_{m}^{q} c_{i}^{\alpha} \frac{\partial \phi_{\alpha}^{m}}{\partial t}\right\}\left[\sum_{\alpha}^{N} \sum_{m}^{q} h\left(\phi_{\alpha}^{m}\right) \frac{\partial c_{i}^{\alpha}}{\partial \mu_{j}}\right]_{i j}^{-1} .
$$

The mobility $\boldsymbol{M}(\phi)$ of the migrating chemical-species, included in the above evolution Eqn. (42) reads

$$
\begin{aligned}
\boldsymbol{M}(\boldsymbol{\phi}) & =\sum_{\alpha=1}^{N} D_{\alpha: i}\left(\frac{\partial c_{i}^{\alpha}}{\partial \mu_{j}}\right) \sum_{m}^{q} h\left(\phi_{\alpha}^{m}\right) \\
& +\sum_{\alpha}^{N} \boldsymbol{D}_{\alpha \alpha: i} \sum_{m<n}^{q, q}\left[\left(\frac{\partial c_{i}^{\alpha}}{\partial \mu_{j}}\right) h\left(\phi_{\alpha}^{m}\right)+\left(\frac{\partial c_{i}^{\beta}}{\partial \mu_{j}}\right) h\left(\phi_{\alpha}^{n}\right)\right] \phi_{\alpha}^{m} \phi_{\alpha}^{n} \\
& +\sum_{\alpha}^{N} \sum_{\alpha<\beta}^{N} \boldsymbol{D}_{\alpha \beta: i} \sum_{m \leq n}^{q, q}\left[\left(\frac{\partial c_{i}^{\alpha}}{\partial \mu_{j}}\right) h\left(\phi_{\alpha}^{m}\right)+\left(\frac{\partial c_{i}^{\beta}}{\partial \mu_{j}}\right) h\left(\phi_{\beta}^{n}\right)\right] \phi_{\alpha}^{m} \phi_{\beta}^{n},
\end{aligned}
$$

where the kinetic coefficients of flux along all possible grain boundaries are included through diffusivity and susceptibility $\left(\partial c_{i}^{\alpha} / \partial \mu_{j}\right)$ matrix.

\subsection{Comparing continuum- and multiphase-field model}

As introduced earlier, a fundamental difference between the continuum- and multiphasefield approach stems from the treatment of the corresponding scalar variables. While a physical attribution in the form of local volume-fraction is imposed on phase-field, the order parameter is treated as an independent variable in continuum-field model. This difference in the consideration of the scalar variables consequently leads to other notable disparities between the two treatments. One such difference is the minimas of the grains (i.e, phase-field/order parameter).

The coupling terms involved in Eqn. (3) of the continuum-field approach, that relate concentration and the order parameter, are written as

$$
\begin{aligned}
& f_{\alpha}\left(c, c_{\mathrm{eq}}^{\alpha}, c_{\mathrm{eq}}^{\beta}, \eta_{i}^{\alpha}\right)=-\frac{m^{\alpha}}{2}\left[\left(c-c_{\mathrm{eq}}^{\beta}\right)^{2}-\left(c-c_{\mathrm{eq}}^{\alpha}\right)^{2}\right]\left|\eta_{i}^{\alpha}\right|^{2}+\frac{n^{\alpha}}{4}\left|\eta_{i}^{\alpha}\right|^{4} \\
& f_{\beta}\left(c, c_{\mathrm{eq}}^{\alpha}, c_{\mathrm{eq}}^{\beta}, \eta_{i}^{\beta}\right)=-\frac{m^{\beta}}{2}\left[\left(c-c_{\mathrm{eq}}^{\alpha}\right)^{2}-\left(c-c_{\mathrm{eq}}^{\beta}\right)^{2}\right]\left|\eta_{i}^{\beta}\right|^{2}+\frac{n^{\beta}}{4}\left|\eta_{i}^{\beta}\right|^{4},
\end{aligned}
$$

where $m^{\alpha}$ and $n^{\alpha}$ are phenomenological constants [33]. Adopting the terms in Eqn. (44) for defining the overall energy-density of the system, the resulting degenerate minimas, following 
Ref. [57], can be written as

$$
\begin{aligned}
& \left\{\eta_{1}^{\alpha}, \eta_{2}^{\alpha}, \cdots, \eta_{q_{\alpha}}^{\alpha}\right\},\left\{\eta_{1}^{\beta}, \eta_{2}^{\beta}, \cdots, \eta_{q_{\beta}}^{\beta}\right\}= \\
& \left\{\left( \pm \Lambda^{\alpha}\left(c_{\mathrm{eq}}^{\alpha}-c_{\mathrm{eq}}^{\beta}\right), 0,0, \cdots, 0\right),\left(0, \pm \Lambda^{\alpha}\left(c_{\mathrm{eq}}^{\alpha}-c_{\mathrm{eq}}^{\beta}\right), 0, \cdots, 0\right), \cdots\left(0,0, \cdots, \pm \Lambda^{\alpha}\left(c_{\mathrm{eq}}^{\alpha}-c_{\mathrm{eq}}^{\beta}\right)\right)\right\} \\
& \left\{\left( \pm \Lambda^{\alpha}\left(c_{\mathrm{eq}}^{\alpha}-c_{\mathrm{eq}}^{\beta}\right), 0,0, \cdots, 0\right),\left(0, \pm \Lambda^{\alpha}\left(c_{\mathrm{eq}}^{\alpha}-c_{\mathrm{eq}}^{\beta}\right), 0, \cdots, 0\right), \cdots\left(0,0, \cdots, \pm \Lambda^{\alpha}\left(c_{\mathrm{eq}}^{\alpha}-c_{\mathrm{eq}}^{\beta}\right)\right)\right\}
\end{aligned}
$$

180 where $\Lambda^{\alpha}=\sqrt{\frac{m^{\alpha}}{n^{\alpha}}}$. However, in the multiphase-field approach, the constraint $\sum_{\alpha} \sum_{i} \phi_{\alpha}^{i}=$ 1 , along with the characteristic property of the interpolation function, $\partial h\left(\phi_{\alpha}^{m}\right) /\left(\partial \phi_{\alpha}^{m}\right)=0$, invariably renders a single minima at 0 .

Preliminary attempts to model multiphase microstructural evolution through continuumfield approach treat concentration as a continuous variable [32, 33]. This consideration is evident in Eqns. (2) and (3). In contrast, recent continuum-field models define their bulk contributions through phase-dependent concentrations [34, 38, 39], and adopt local equilibrium condition in the diffuse interface region [51, 52], as expressed in Eqn. (25), through appropriate interpolation function [40]. This treatment of the concentration efficiently decouples the bulk and interface contributions, and furthermore, results in the minimas

$$
\begin{aligned}
& \left\{\eta_{1}^{\alpha}, \eta_{2}^{\alpha}, \cdots, \eta_{q_{\alpha}}^{\alpha}\right\},\left\{\eta_{1}^{\beta}, \eta_{2}^{\beta}, \cdots, \eta_{q_{\beta}}^{\beta}\right\}= \\
& \{( \pm 1,0,0, \cdots, 0),(0, \pm 1,0, \cdots, 0), \cdots(0,0, \cdots, \pm 1)\} \\
& \{( \pm 1,0,0, \cdots, 0),(0, \pm 1,0, \cdots, 0), \cdots(0,0, \cdots, \pm 1)\}
\end{aligned}
$$

which are independent of any phenomenological and material constants [57]. Despite the simplification of the minimas, this continuum-field model yields numerous degenerate minimas, in contrast to the multiphase-field model.

As opposed to the seemingly conventional Landau-type polynomial, the concentration-based energy density in continuum-field treatment, which encompasses $k$-component [34], is expressed following a numerical approach originally intended to circumvent the formation of spurious phases in multiphase-field model [58]. Accordingly, this bulk contribution reads

$$
f_{\mathrm{ch}}=\frac{\bar{A}}{2} \sum_{i=1}^{k-1}\left[c_{i}-\sum_{\alpha}^{N} c_{i: \mathrm{eq}}^{\alpha} H_{\alpha}\left(\eta^{\alpha}\right)\right]^{2}+\sum_{\alpha}^{N} \bar{B}^{\alpha} H_{\alpha}\left(\eta^{\alpha}\right)
$$


where $\bar{A}, \bar{B}^{\alpha}$ and $H_{\alpha}$ pertain to the fitting constants and interpolation function of the multicomponent continuum-field model, respectively. Although the energy density in Eqn. (47) facilitates in preserving phase-fractions, given the phase- and component-independent nature of the prefactor $\bar{A}$, it is arguable that this formulation can be adopted to incorporate quantitative CALPHAD-based data. Moreover, since the curvature-driven transformation kinetics in higherorder systems, ternary and above, is influenced by the second-derivative of the free-energy function [59, 60], an independent prefactor can unfavourably influence the kinetics. While following similar polynomial approximation, in the current multiphase-field treatment, the bulk contribution is made conducive for incorporating quantitative data through phase- and componentdependent fitting parameters [61]. For an elaborate discussion on the free-energy density formulation for multicomponent systems, and the introduction of CALPHAD data, the readers are directed to Refs. [62, 27] (previous works of the authors).

Additionally, when compared to the present approach, the profile of the scalar variable across the interface is significantly different from the existing continuum models. This is primarily due to the nature of the energy-density formulation.

\subsection{Domain configuration}

Concurrent grain growth and coarsening exhibited by duplex and triplex microstructures of binary and ternary systems in two- and three-dimensional setup is comparatively analysed in the present work. Irrespective of the dimension, the domain is discretised into cells of unit dimensions, $\Delta \mathrm{x}=\Delta \mathrm{y}(=\Delta \mathrm{z})=1.0$ (non-dimensionalised). The temporal evolution of phase-field and chemical-potential, in Eqns. (27) and (42), are solved over the homogeneous cells through forward-marching Eulers scheme. A definite width is assigned to the diffuse interface by affixing the length scale parameter at $\varepsilon=4 \Delta \mathrm{x}$. Furthermore, periodic boundary condition is imposed along all the terminations of the two- and three-dimensional domains. The computational resource is efficiently consumed by Locally Reduced Order Parameter (LROP) optimization, which ensures that only required phase-fields are solved locally, and Message Passing Interface (MPI).

For all two-dimensional simulations, a domain of size $2048 \times 2048$ cells is adopted, which renders around 10000 grains. Almost similar number of grains in three-dimension are devised in 
Table 1: List of simulation parameters

\begin{tabular}{c|c|c} 
Parameter & symbol & value \\
\hline Grid size & $\Delta \mathrm{x}=\Delta \mathrm{y}(=\Delta \mathrm{z})$ & 1.0 \\
Time-step width & $\Delta \mathrm{t}$ & 0.02 \\
Interface-width parameter & $\varepsilon$ & $4 \times \Delta \mathrm{x}$ \\
Relaxation parameter & $\tau$ & 1.0
\end{tabular}

a domain of $300 \times 300 \times 300$. Owing to the comparative nature of the present analysis, dimensionless time and time step ( $t$ and $\Delta t$, respectively) is considered for monitoring and discussing the evolution.

Independent of the ultimate nature of the microstructure, single phase or otherwise, Voronoi tesselation is adopted to establish a polycrystalline system in the domain, two- or three-dimension. Subsequently, the randomly distributed grains are distinguished into phases by assigning equilibrium concentrations, and imposing corresponding energy-density. In order to ensure that the desired phase-fraction is achieved, appropriate number of grains for each phase are conscientiously selected. The bulk contribution which accompanies the equilibrium composition ensures that the overall volume-fraction of the phases are preserved during the microstructural transformation. The parameters involved in this initialisation of the domain is listed in Table 1

\section{Results and discussions}

\subsection{Influence of number of phases and components}

\subsubsection{Microstructural evolution}

Regular grain-growth in a chemically-homogeneous system can be modelled by exclusively considering the interface contribution. Therefore, in the present approach, the regular graingrowth is simulated by overlooking the role of the bulk energy-densities. The microstructural evolution of the chemically-identical grains is shown in Fig. 1

The progressive decrease in the overall number of grains with the gradual increase in the size is evident from this illustration. Temporal change in the duplex and triplex microstructures of 

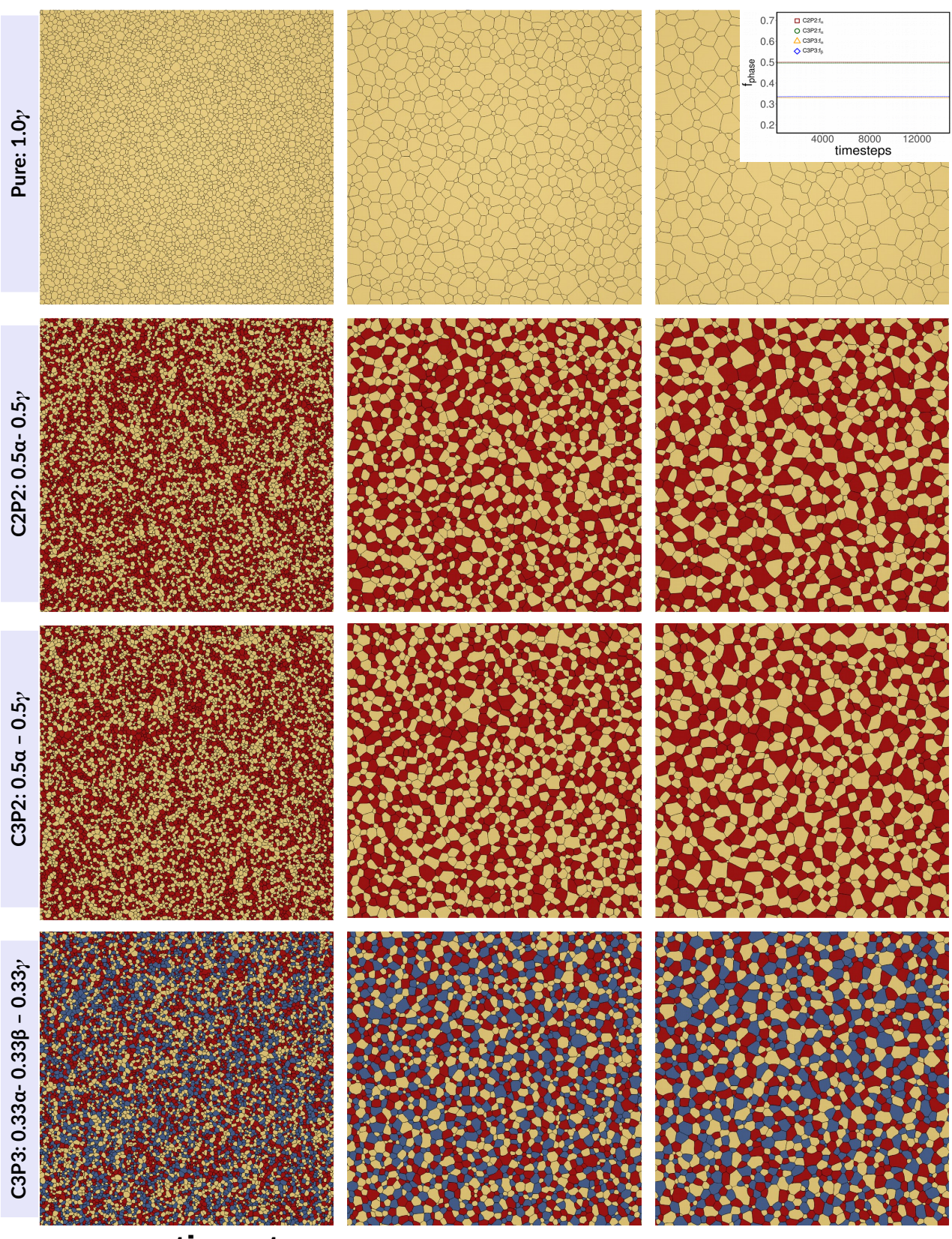

timesteps

Figure 1: Microstructural evolution of single-, two- and three-phase polycrystalline structures indicated as 'Pure', 'C2P2', and 'C3P', respectively. Two duplex microstructures of binary (C2P2) and ternary (C3P2) systems are considered for analysing the effect of number of components. 
Table 2: List of material parameters

\begin{tabular}{c|cc|c} 
Parameter & \multicolumn{2}{|c|}{ symbol } & value \\
\hline Grain boundary energy & $\gamma_{\Theta \Theta}$ & $\Theta \in\{\alpha, \beta, \gamma\}$ & 1.0 \\
Interphase energy & $\gamma_{\Theta \delta}$ & $\Theta, \delta \in\{\alpha, \beta, \gamma\}$ & 1.0 \\
Bulk diffusivity & \multicolumn{2}{|c|}{$D^{\alpha}=D^{\beta}\left(=D^{\gamma}\right) \equiv D$} & 1.0
\end{tabular}

respective two- and three-components system are included in Fig. 1 Moreover, to understand the changed all-through the evolution, owing to its importance, the volume-fractions of the importance phases are monitored and plotted. The time-invariant behaviour of the volume-fraction curve in Fig. 1] definitively attests to the preservation of phase-fraction, despite the noticeable change in the distribution. 
Table 3: Equilibrium concentration of binary and ternary systems

\begin{tabular}{c|c|c} 
Phase & Independent component $-i$ & Independent component- $j$ \\
\hline (Binary) & & - \\
Phase- $\alpha$ & 0.1 & - \\
Phase- $\beta$ & 0.9 & \\
\hline (Ternary) & & 0.05 \\
Phase- $\alpha$ & 0.05 & 0.9 \\
Phase- $\beta$ & 0.05 & 0.05 \\
Phase- $\gamma$ & 0.9 &
\end{tabular}

\subsubsection{Decrease in number of grains}

The incremental change in the number of grains, in all the difference microstructures considered, is tracked and plotted in Fig. 2. Rate of change in the total number of grains can be considered as a parameter to realise the kinetics of grain growth. Accordingly, Fig. 2 indicates that the rate of evolution is noticeably high single-phase homogeneous, followed by duplex microstructure, with three-phase system exhibiting relatively low-rate of evolution.

Moreover, in all systems, including single-phase, the change in total number of grains with time adheres to a similar trend, reflecting the aspect of grain growth which prevails in multiphase microstructures as well. This behaviour is primarily attributed to the geometrical and topological factors that dictate the evolution of a grain. In Fig. 2 it is interesting to note that duplex microstructures of binary and ternary system transform at a comparable rate. In other words, while increasing the number of phases visibly decreases the grain-growth kinetics, the increase in the number of components does not render an analogous effect. However, a marginal disparity is noticeable in Fig. 2, which indicates that grains in ternary duplex-system disappear in a relatively lower rate than the corresponding binary system. The influence of the number of phases and chemical components on the kinetics of evolution is definitively assessed by considering the change in average grain-size. 


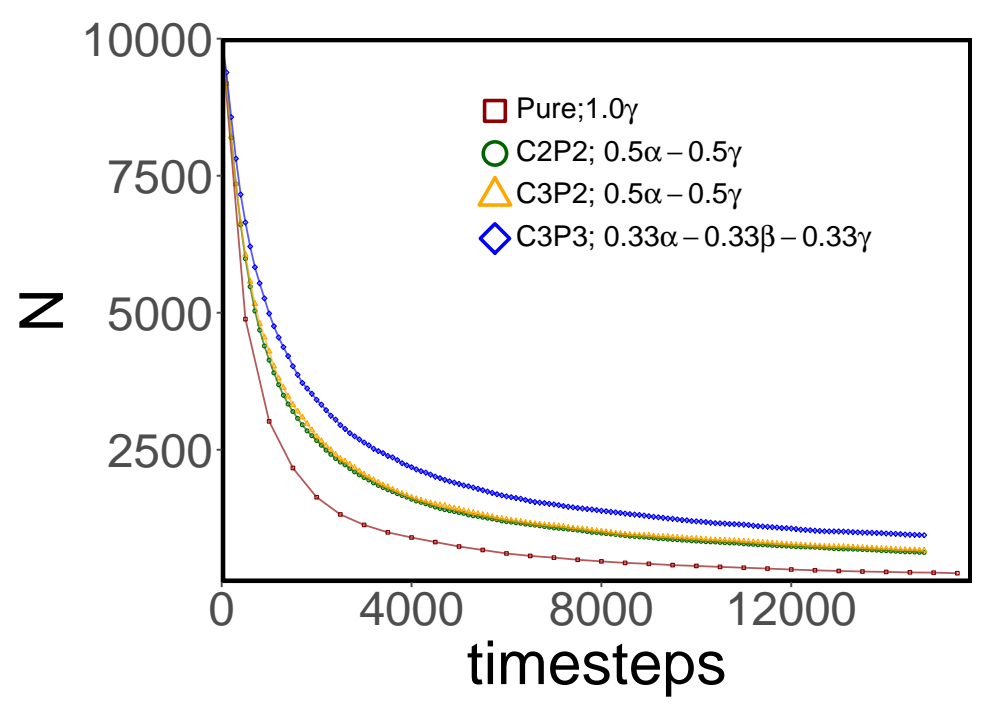

Figure 2: Monotonic decrease in the total number of grains during the evolution of single-phase, duplex and triplex microstructures of binary and ternary systems.

\subsubsection{Transformation kinetics}

The progressive increase in the average grain-size with time for single- and multiphase microstructures are collectively plotted in Fig. 3 . While the exponent term $m$ is equal to 2 in the chemically homogeneous system, the microstructural changes in duplex and triplex microstructure yields $m \approx 3$. This difference in the exponent value is due to the disparity in the mechanism governing the grain growth. In the single-phase system, the microstructural changes are dictated by the migration of the interface, which correspondingly yields a exponent value of $m \approx 2$. However, the exponent value of 3 , in two- and three-phase system indicates a diffusion-governed grain growth. These differences in the exponent terms are consistent with the existing experimental [63, 64] and theoretical results [32, 33, 38].

In compliance with the temporal change in total grain, Fig. 1, the average grain-size plot in Fig. 3 indicates that with increase in number of phases, the transformation kinetics significantly decreases. Moreover, the duplex microstructures of binary and ternary system show a marginal difference, with two-component system exhibiting a slightly higher growth rate. The trend in Fig. 3, which shows that grain growth is faster in single-microstructure, respectively followed by duplex and triplex system, assert that the transformation is noticeably stunted due to the 


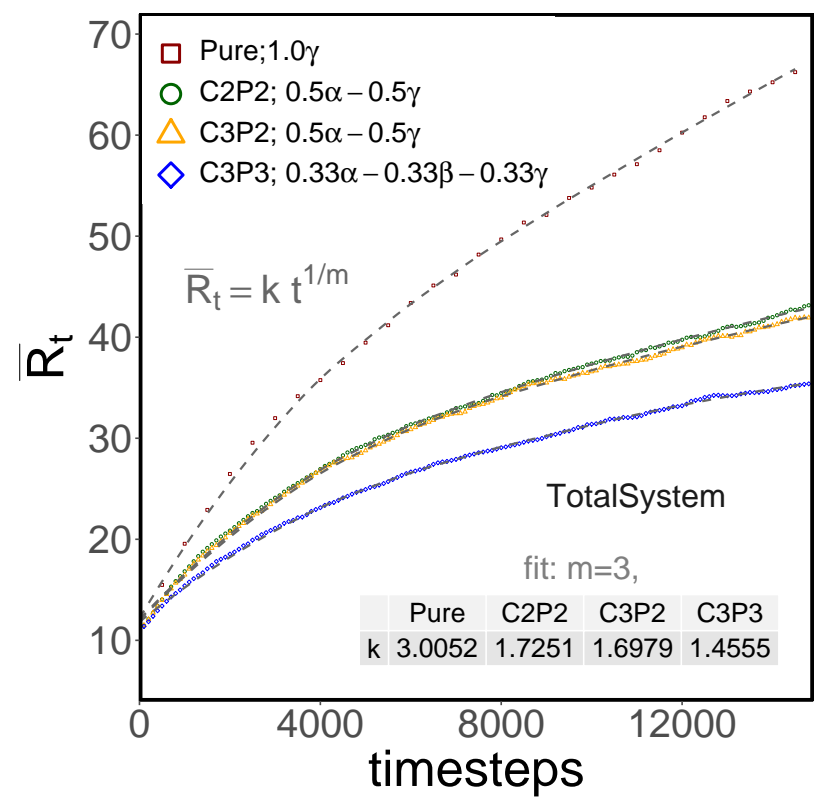

Figure 3: Temporal increase in the average grain size of the polycrystalline systems with chemically-identical and phase-associated grains during the microstructural transformations.

increase in the number of phases. However, similar influence is not rendered by the number of chemical species.

The inverse relation between the number of phases and growth kinetics can be attributed to the phase distribution. From Fig. 3 it is evident that the transformation in multiphase microstructure is dictated by diffusion. While this difference in the transformation mechanism is responsible for disparity in the single- and multiphase system, a similar argument can be made for the lower growth rate triplex microstructure. Diffusion-governed microstructural transformation in multiphase polycrystalline system depends on the distribution of the phases. In other words, the transformation rate of two isolated chemically-identical grains proportionately varies with the distance separating them. The introduction of a new phase substantially convolutes the arrangement of the phases, and consequently, the diffusion path gets increasingly complex, thereby decreasing the overall kinetics of grain growth. The effect of components is not as straightforward as the number of phases, therefore, the growth rate in duplex microstructures of binary and ternary system are separately analysed. 


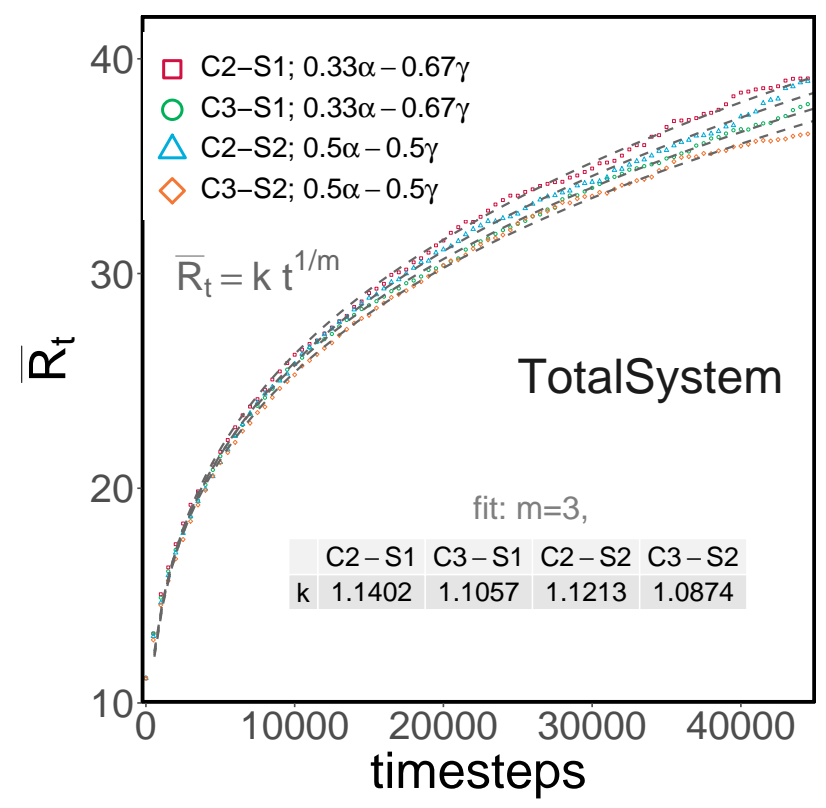

Figure 4: Change in the average grain size of duplex microstructure with equal and unequal phase-fraction pertaining to binary and ternary systems with time. The binary and ternary duplex microstructures with equal phase-fractions are correspondingly represented by 'C2-S2' and 'C3-S2', while 'C2-S1' and 'C3-S1' respectively denotes the duplex systems with unequal volume-fractions of phases.

\subsubsection{Duplex microstructures of binary and ternary system}

Increase in the average grain-size with time during the microstructural evolution of twophase system with two and three components are presented in Fig. 4. As opposed to the cumulative depiction with single and three-phase microstructures in Fig. 3 the difference in the grain growth kinetics introduced by an additional component is evident in Fig. 4

Although this separate illustration unravels a perceivable disparity in the rate of change in average grain-size between binary and ternary system with duplex microstructure, the effect is relatively less definite when compared to the number of phases. In order to ensure that the minimal decrease in the transformation kinetics is not due to the equal volume-fractions of the phases, another setup with a minor and major phase distribution is considered.

A microstructure with unequal phase-fraction, characterised by $33 \%$ phase- $\alpha$, is devised to substantiate the understanding on the effect of number of components. The equilibrium concentrations, and the bulk contributions, are appropriately assigned to configure a binary and 


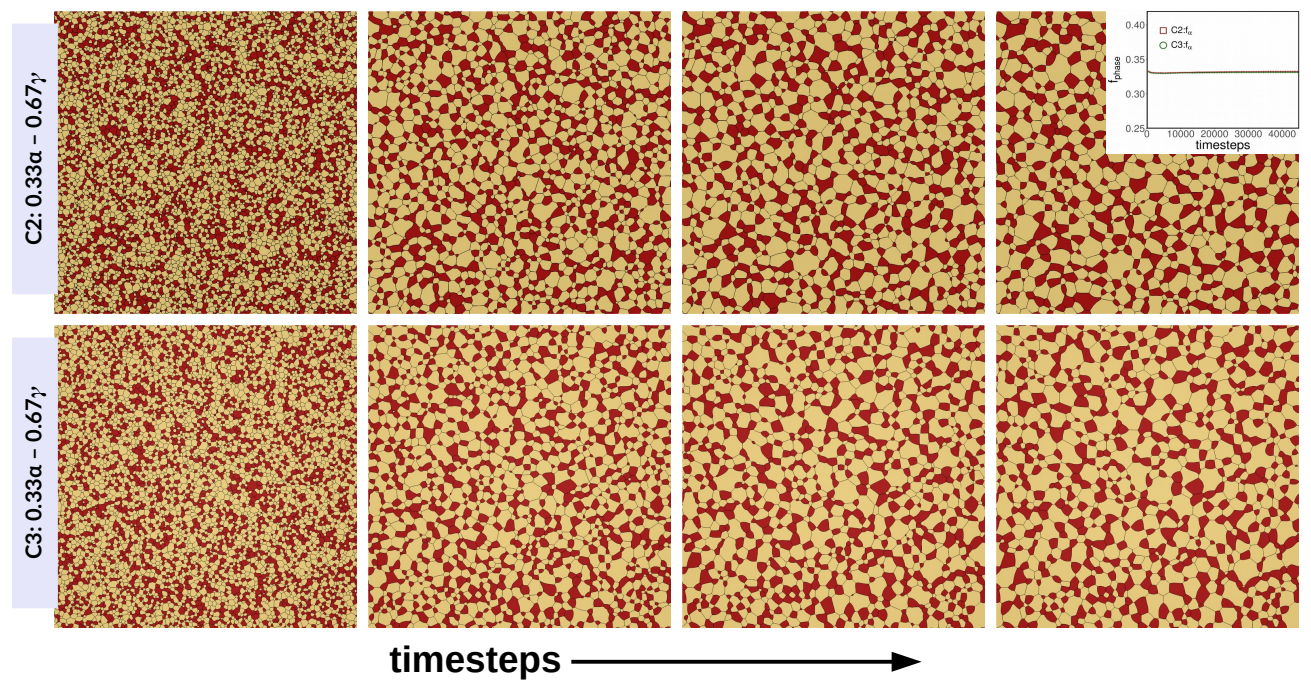

Figure 5: Concurrent grain growth and coarsening of binary and ternary duplex microstructures with unequal phase-fractions.

ternary system based on this microstructure. The two- and three-component duplex microstructures with minor phase- $\alpha$ is allowed to evolve towards a decrease in the overall energy-density of the system. Change in the average grain-size with time, which accompanies the evolution of the binary and ternary two-phase microstructures, is collectively plotted in Fig. 5.

The concurrent grain-growth and coarsening exhibited by these systems of unequal phasefractions is included as a subset. Analogous to the other evolutions illustrated in Fig. 1, microstructures with different volume-fraction of the phases adheres to a continuous distribution all-through the transformation. More importantly, the growth kinetics in the ternary system is relatively lower than the corresponding two-component microstructure. This difference introduced by the additional components is comparable to its influence on the microstructure with equal phase-fraction (4), and is not significant. Before delineating the reason for the minimal change in the transformation kinetics with increase in number of components, the growth rate exhibited by individual phases in binary and ternary duplex microstructures of unequal phasefraction is studied.

In Fig. 6, the increase in the average grain-size of phase- $\alpha$ and $-\gamma$ during the evolution of the two- and three-component microstructures with different phase-fraction is shown. This il- 


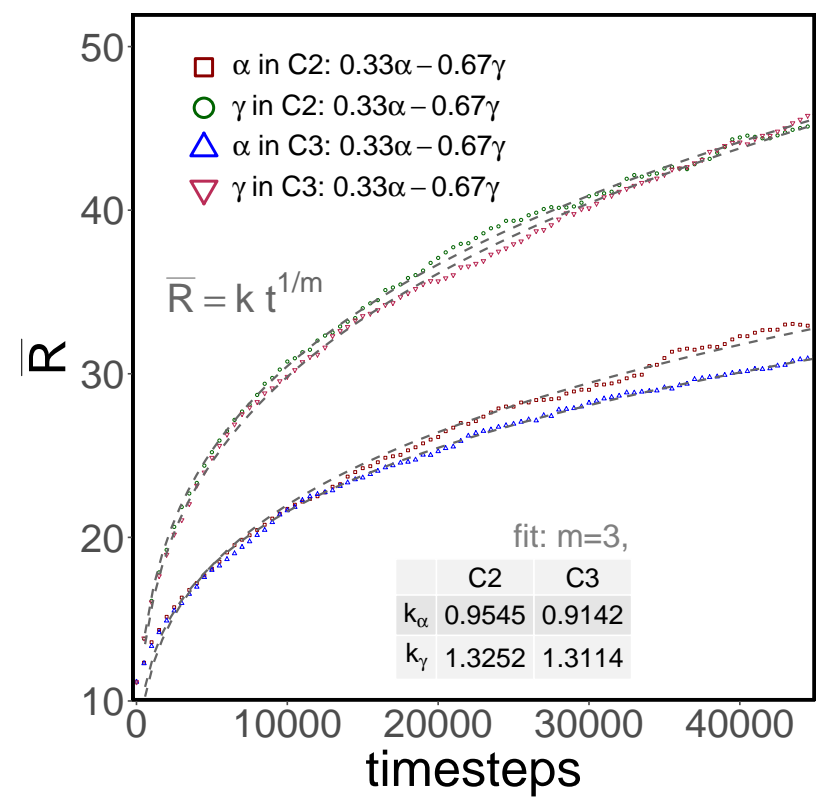

Figure 6: Grains of the binary and ternary duplex microstructures are distinguished based on the phases and the increase in its average size during the transformation is plotted with time.

lustration unravels that, irrespective of chemical-makeup of the system (binary or ternary), the growth rate of the minor phase- $\alpha$ is visibly lower than the corresponding phase- $\gamma$. The sluggish growth of the minor phase is consistent with the existing studies, and is primarily due to the increase in the distance separating the chemically-similar $\alpha$-grains, which consequently prolongs the time taken for the diffusion of the components. Interestingly, in the microstructure of unequal phase-fraction, the effect of the additional component is more definite in the minor phase, when compared to phase- $\gamma$. In other words, it is evident from Fig. 6 that the growth rate of phase- $\alpha$ is reduced in ternary system, while the evolution of the major phase remains undisturbed by the inclusion of an additional component.

Irrespective of the degree of disparity, the transformation kinetics exhibited by the ternary duplex-microstructure is different from the corresponding binary setup. This decrease in the growth rate of three-component microstructure is due to the convolution of the concentration evolution. While the increase in the number of phases disturbs the diffusion path, the addition of a component increases the number of factors governing the migration of the chemical species. In a binary system with one independent component, the diffusivity, and the proportionality 
constant, which relates it to the mobility, assume a scale value. These kinetic-coefficients are no-longer scalar parameters in a ternary system, but include the contribution of interdiffusion through appropriate matrices. Therefore, the concentration evolution in ternary setup gets intricate, when compared to binary, due the inter-dependencies of the migrating chemical-species. This convoluted evolution of the components ultimately leads to the difference in the transformation kinetics between the two- and three-component multiphase systems. However, the equal kinetic-coefficients considered in the current investigations minimises the difference introduced by the additional component.

\subsection{Influence of diffusivity}

Although increase in the number of components convolutes the concentration evolution, its effect on the growth rate is restricted by the kinetic coefficients. To explicate the role of diffusivity in the transformation kinetics, the microstructural changes governed by different diffusion coefficients are comparatively analysed. In Fig. 7, the temporal change in the average grainsize accompanying the evolution of binary duplex-microstructure with independent diffusivity $D_{i}=0.5$ and $D_{i}=0.1$ is illustrated.

Despite the equal number of components, the disparity in the diffusion coefficient profoundly effects the kinetics of evolution. The system with higher diffusivity evolves at a much faster rate when compared to the microstructure associated with lower kinetic coefficient. With time, as shown in Fig. 7 the difference in the kinetics gets increasingly significant. This behaviour is primarily due to the progressive change in the distribution of the phases.

As the grains of the two-phase microstructure grow, owing to the prevailing continuous distribution, the distance separating the chemically-identical grains increases. Consequently, the distance traveled by the migrating species to establish a microstructural change proportionately increases. With the expansion of the diffusion pathways, the influence of the diffusion coefficient gets more defined, which in-turn renders an increasing disparity in the kinetics of evolution, as observed in Fig. 8

A ternary duplex-microstructure with diffusivity of one independent component similar to the binary system of $D_{i}=0.5$ is allowed transform. The increase in the average grain-size with 


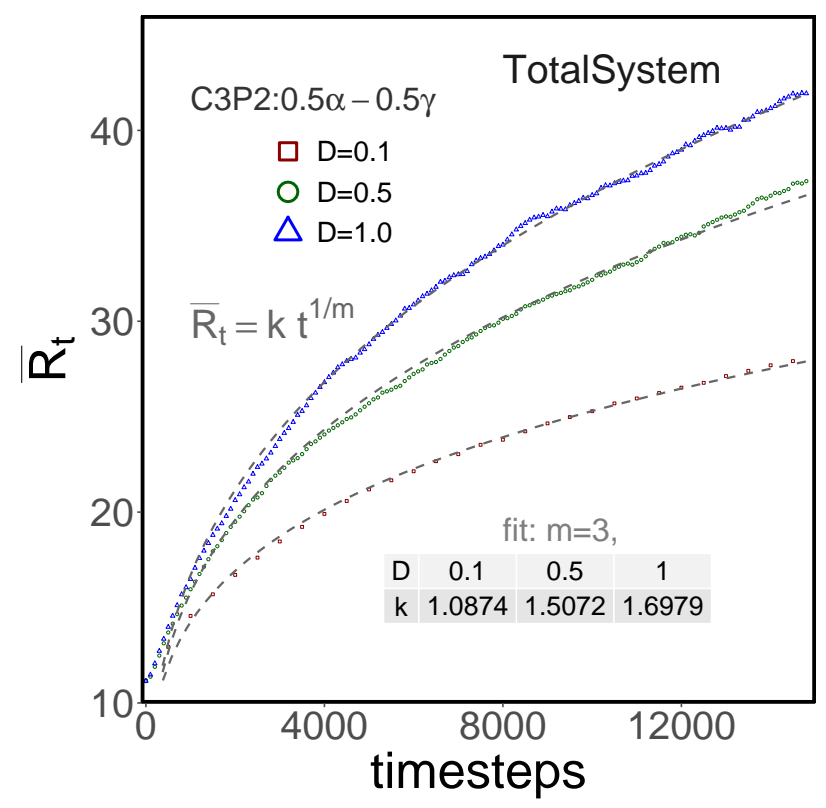

Figure 7: The disparity in the rate of increase in average grain size of ternary duplex microstructure with equal phase-fraction due to the difference in bulk diffusivity of the phases.

time during this evolution is included in Fig. 8

When compared to the binary system of lower diffusivity, the growth rate in the ternary microstructure, with $D_{i}=0.5$, is noticeably higher. Furthermore, the transformation kinetics of binary and ternary duplex-system with identical diffusion coefficient exhibit only a marginal disparity, with three-component microstructure evolving at a slightly lower rate. Accordingly, Fig. 8 unravels that the influence of components is made definitive exclusively through the kinetic coefficients, while the increase in number of chemical species alone, though convolutes the diffusion mechanism, offers a restricted disparity.

\subsection{Evolution of two-dimensional triplex microstructure}

\subsubsection{Microstructural transformation}

The microstructural transformation of ternary three-phase microstructures is examined to understand the influence of the volume-fractions of the phases on the kinetics of the evolution. In Tables. 1. 2 and 3 the parameters associated with the modelling three-phase system is presented. Three different microstructural setups with varying phase-fractions are considered. In Fig. 9 , the 


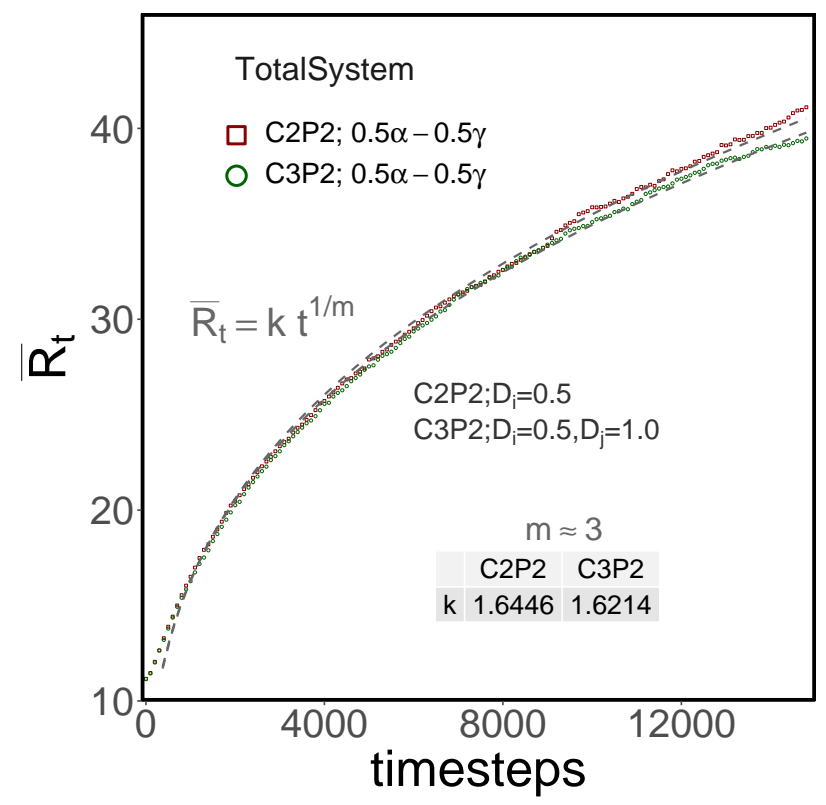

Figure 8: The marginal difference in the transformation kinetics of binary and ternary duplex systems with an independent component- $i$ of identical diffusivity.

temporal evolution of these triplex microstructures with equal, $33 \% \alpha-33 \% \beta$, and unequal, $25 \% \alpha$ $-25 \% \beta$ and $8 \% \alpha-8 \% \beta$, phase-fraction is illustrated.

Owing to the consideration of equal grain boundary and inter-phase energy densities, the entire evolution is devoid of any clusters of a particular phases. In contrast, a continuous and random arrangement of the phase-associated grains is evident in Fig. 9

\subsubsection{Transformation kinetics}

The kinetics of the microstructural transformation of the various ternary triplex-systems is ascertained by monitoring the change in the average grain-size. Increase in the average grainsize of different three-phase microstructures with time is collectively plotted in Fig. 10 The setup with least fraction of the minor phases, $8 \% \alpha-8 \% \beta$, exhibits a higher growth rate, while the transformation kinetics in the microstructure with equal phase-fraction is significantly low. Triplex system with unequal phase-fraction characterised by $25 \% \alpha-25 \% \beta$ transforms at a rate marginally greater than equal phase-fraction setup, but noticeably lower than $8 \% \alpha-8 \% \beta$ microstructure. To understand the influence of the phase-fractions on the transformation kinetics, 

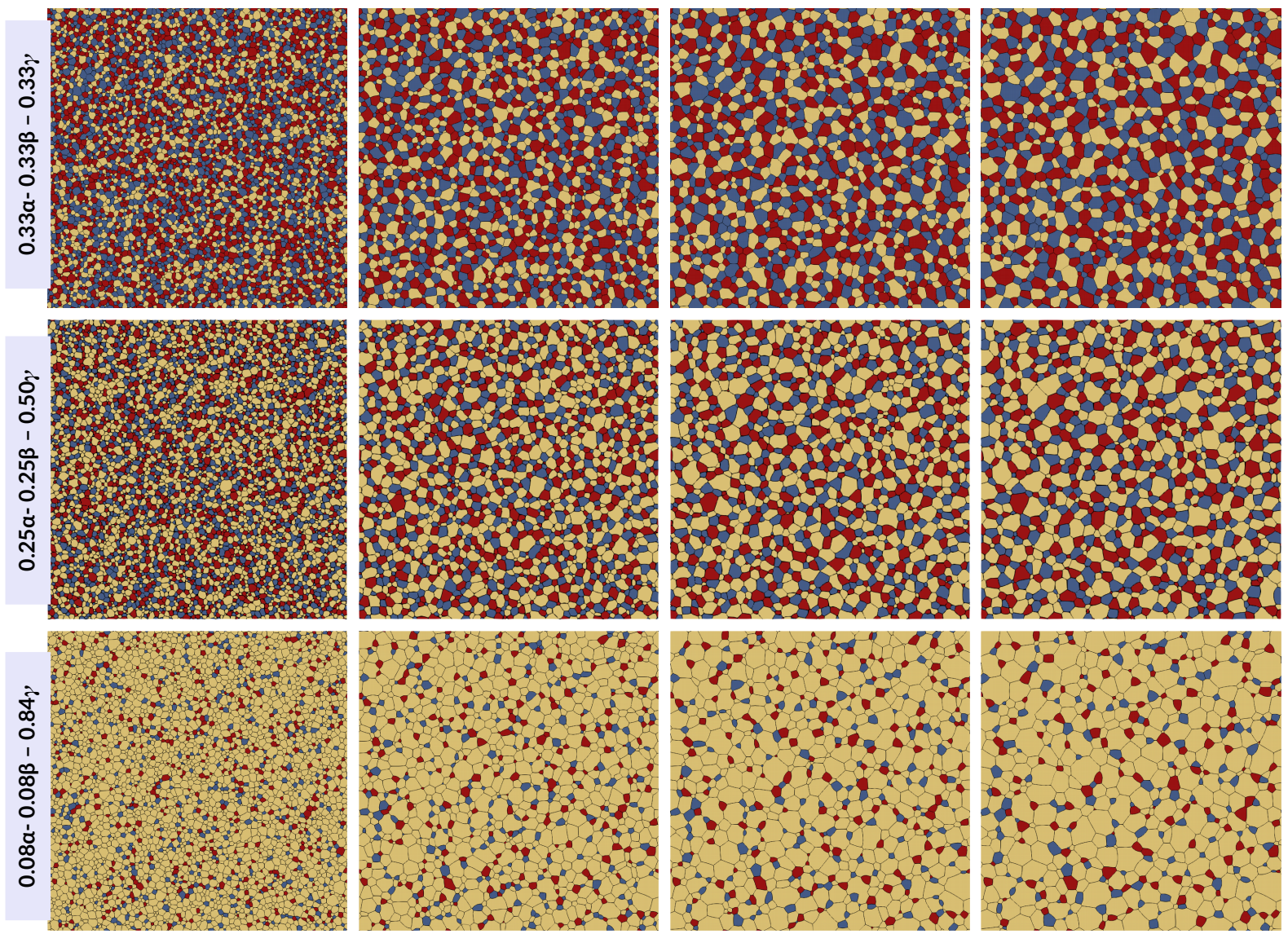

timesteps

Figure 9: Microstructural transformation of ternary three-phase polycrystalline systems with equal and unequal phase-fractions. Two setups with varying volume fraction of the major phase- $\gamma$ is considered to unravel the influence of phase-fraction on evolution kinetics. 


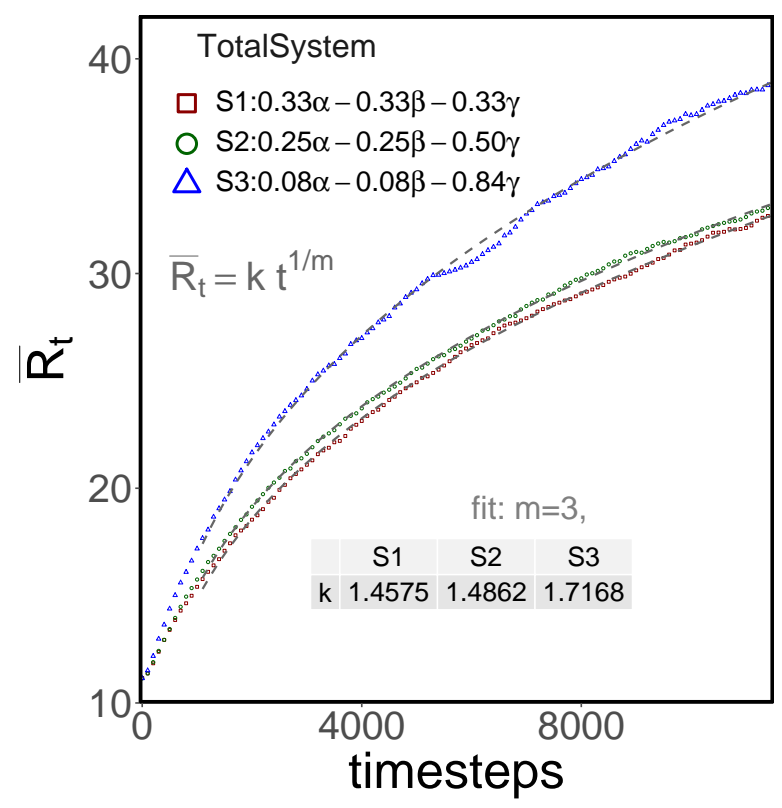

Figure 10: Progressive increase in the average grain size of the ternary triplex microstructures of different phasefractions with time.

the growth rate of the individual phases pertaining to these triplex microstructures are analysed.

Triplex system with unequal phase-fraction characterised by $25 \% \alpha-25 \% \beta$ transforms at a rate marginally greater than equal phase-fraction setup, but noticeably lower than $8 \% \alpha-8 \% \beta$ microstructure. To understand the influence of the phase-fractions on the transformation kinetics, the growth rate of the individual phases pertaining to these triplex microstructures are analysed.

In Fig. 11, the increase in the average grain-size of the phase-associated grains are distinguished based on the chemical makeup, and are collectively presented. Since the volume fraction of the minor phases are equal, only one of the phase $(\alpha)$ is chosen for this illustration. For the triplex microstructure with equal phase-fractions, evolution rate of $\alpha$-grains are plotted in Fig. 11.

Fig. 11 indicates that, in all the triplex microstructures, the minor phase-grains generally exhibit a low rate of transformation when compared to their counterparts. Moreover, the growth rate of $\alpha$-grain in the microstructure with $80 \% \gamma$ is the least of all the phases considered. The minor phase-grains of $25 \% \alpha-25 \% \beta$ transform at a visibly greater rate than the corresponding 


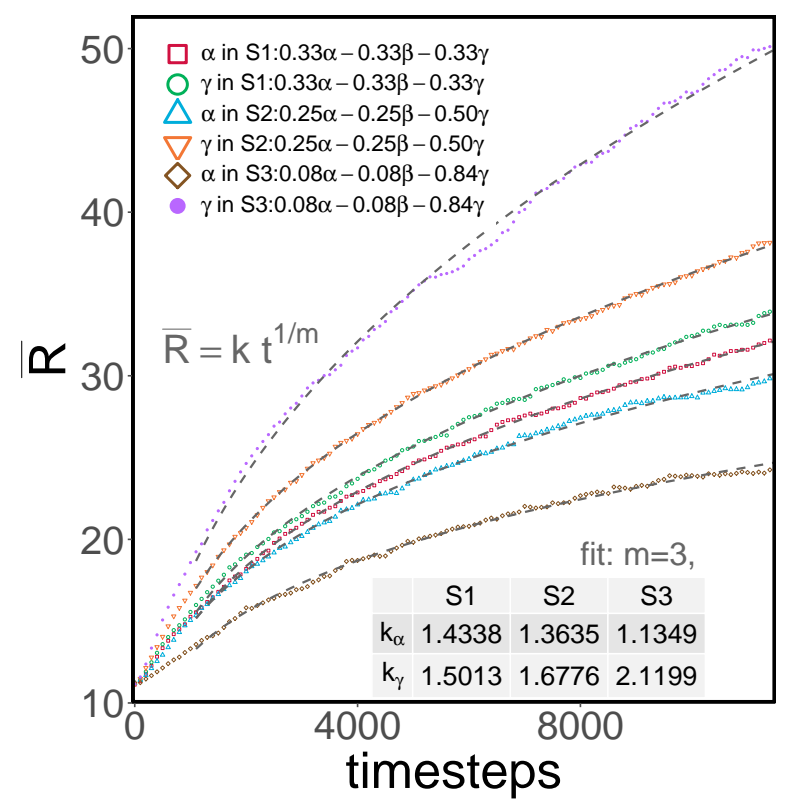

Figure 11: Temporal change in the grain sizes of the ternary triplex-microstructure with equal and unequal phasefractions are plotted by distinguishing the grains based on it chemical composition.

grains in $8 \% \alpha$ in the triplex microstructure. In contrast to the evolution of the minor phases, Fig. 11 shows that the highest transformation kinetics is exhibited by the phase- $\gamma$ grains of $8 \% \alpha$ $-8 \% \beta$ microstructure, which is followed by the major-phase grains of $25 \% \alpha-25 \% \beta$ setup. The growth rate of the system with equal phase-fraction lies in between the major- and minor-phase grains of triplex microstructure with unequal volume fractions.

The trend in Fig. 11 reflecting the influence of phase-fraction on the transformation kinetics of triplex microstructure can be realised by the considering the growth rate of major-phase grains. In minor-phase grains, irrespective of its volume fraction, the evolution is predominantly dictated by the diffusion of the chemical species. Therefore, when its volume fraction decreases, the minor-phase grains get distributed farther apart, ultimately prolonging the time-taken for diffusion. This delay in diffusion considerably stunts the growth of the minor-phase grains. However, in the major-phase grains, the evolution is interplay of diffusion and interface-migration. With considerable increase in the volume fraction of a particular phase-grains, due to the decrease the diffusion length, the interface migration begins to play a dominant role. Correspondingly, the growth rate of the major-phase grains gets exceedingly high. This behaviour of the major- 
and minor-phase grains cumulatively renders the transformation kinetics illustrated in Fig. 11 Moreover, the marginal difference in the growth rate of $25 \% \alpha-25 \% \beta$ and equal phase-fraction microstructure indicate the balance in the diffusion-controlled and interface-governed evolution. However, when the volume fraction of major phase is at $80 \%$, the interface migration begins to dominate the growth rate of the transformation, thereby rendering high transformation kinetics.

\subsection{Three-dimensional evolution of triplex microstructure}

\subsubsection{Microstructural transformation}

Understanding of the microstructural evolution in two-dimension is relevant only to a closerange of system like thin films [65, 66]. In order to extend the applicability of an investigation, the analysis is extended to three-dimensional setups [33]. Therefore, the evolution of the ternary triplex-microstructure is studied in three-dimension. Since the primary focus of this work is to explicate the ability of the present multiphase-field approach to model the three-dimensional multiphase transformation, a representative system of equal phase-fraction is considered.

In Fig. 12 the progressive change in the three-phase microstructure in three-dimensional domain is illustrated. Owing to the nature of the grain boundary and interphase energy-densities, which are assumed to be constant and equal, the triplex microstructure evolves with a continuous distribution of phases without any formation of identical-grain clusters.

The volume fraction of the phases during the transformation is monitored and plotted as a subset in Fig. 12 This depiction indicates that the phase-fraction remains unaltered all-through the three-dimensional transformation of ternary triplex-microstructure. However, as evident in Fig. 12, the number of phase-associated grains continue to decrease during the evolution inkeeping with grain-growth aspect of the transformation.

\subsubsection{Transformation kinetics}

The temporal increase in the average grain-size of the three-dimensional triplex microstructure during the concurrent grain growth and coarsening is present in Fig. 13 . Since a system of equal phase-fraction is considered, the depiction illustrating the kinetics of evolution is not dissociated for individual phases. Consistent with the mechanism governing the transforma- 


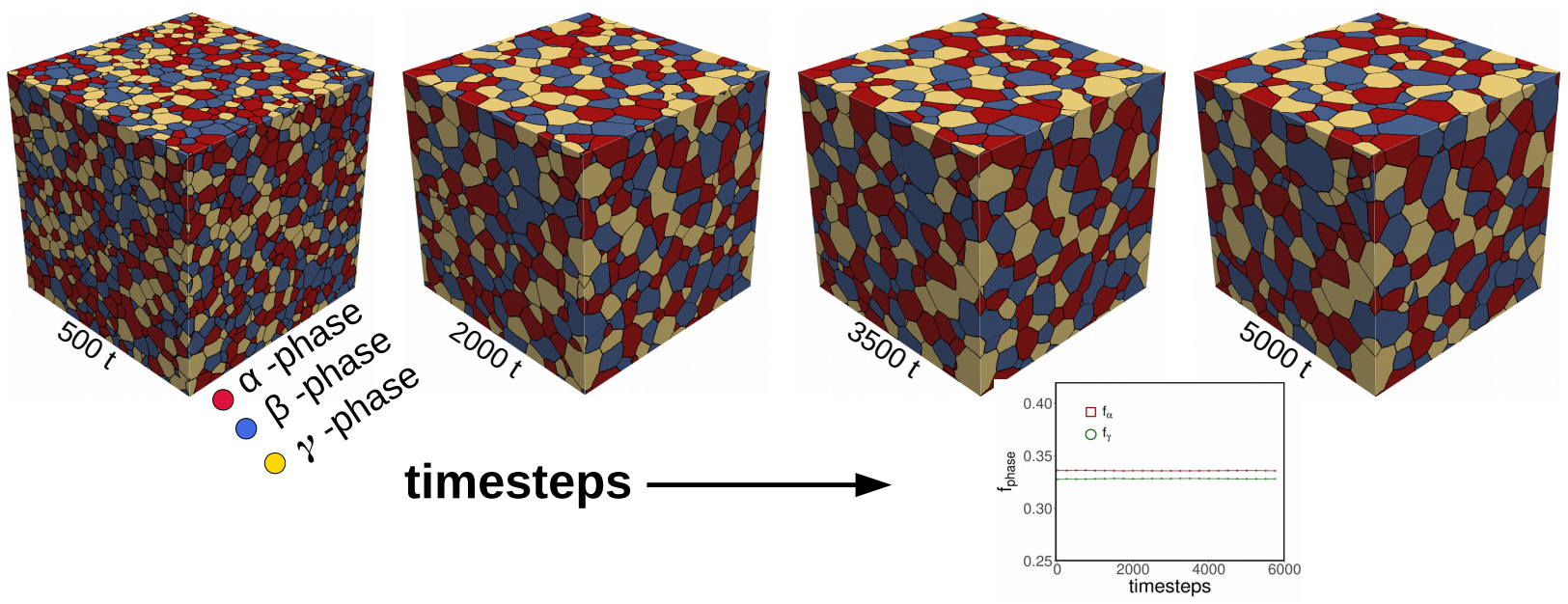

Figure 12: Concurrent grain growth and coarsening of ternary triplex-microstructure with equal volume-fraction of the constituent phases in three-dimension. The volume fraction of the individual during the evolution is monitored and presented as a subplot.

tion, and the existing works [34, 39], the exponent $m$ is realised to be close to 3 in the current three-dimensional transformation of the ternary triplex-microstructure.

For comparison, the change in the average grain-size of two-dimensional triplex setup of equal phase-fraction with time is included in Fig. 13. The growth rate of the triplex system is significantly higher in three-dimension where compared to the corresponding two-dimensional setup. This disparity in the transformation kinetics between the similar microstructures in different dimensions can be attributed to the increased amount of interfacial area, and additional degree of freedom for evolution.

\subsubsection{Grain-size distribution}

The distribution of the grain size in two- and three-dimensional setup of triplex microstructures at three difference timesteps during the transformation is shown in Fig. 14 . The sizes of the grains for this distribution plot are normalised through the critical grain-size. Different relations are employed to determine the critical grain-size of the two- and three-dimensional microstructure [67]. Irrespective of the dimension, both three-phase microstructures reach a time-invariant size as the evolution proceeds.

However, Fig. 14 unravels that the time-invariant distribution established in three-dimensional 


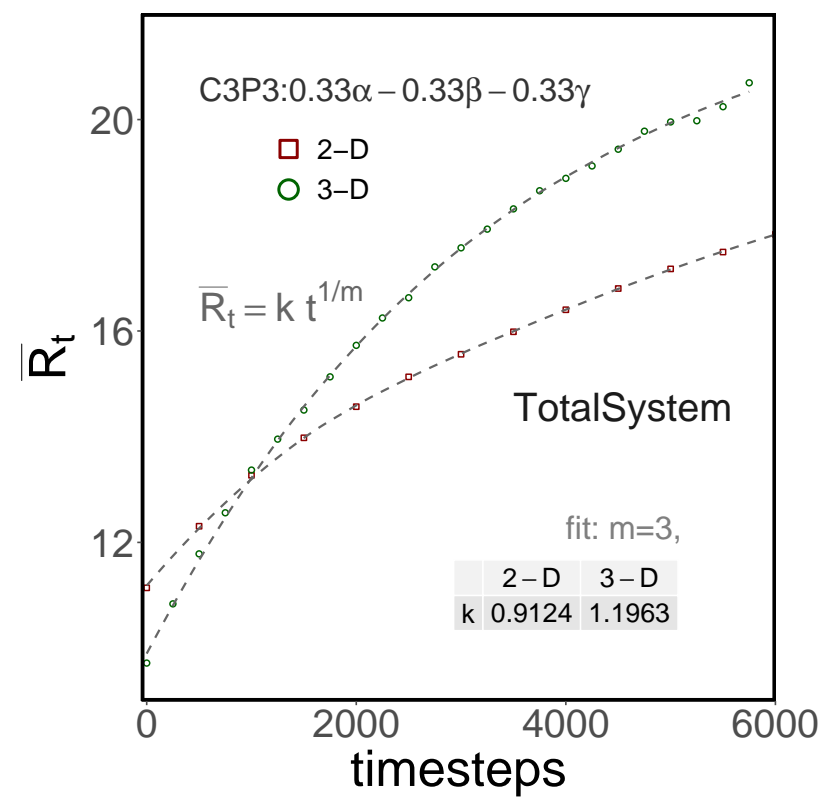

Figure 13: Change in the average grain size of ternary triplex-microstructure in two- and three-dimension are collectively plotted with time for a comparison.

system is significantly different from the respective distribution in two-dimensional triplex microstructure [67, 68, 69]. In other words, the grain-size distribution, in three-dimension, is confined to $\frac{R}{R_{\mathrm{cr}}}=2$, whereas the tail of the size distribution plot pertaining to two-dimensional three-phase system smoothly extends beyond this critical ratio. Apparently, while the ternary triplex-microstructure adheres to Hillert distribution in three-dimension, in two-dimension, the distribution deviate from it by extending beyond $\frac{R}{R_{\mathrm{cr}}}=2$ [67]. In Fig. 14, the grain-size distribution of the two-dimensional triplex microstructure follows Weibull distribution of shape parameter, $[\beta=2.70]$.

\section{Summary}

Theoretical analysis of grain growth have largely been confined to polycrystalline systems wherein the grains are chemically homogeneous. Such consideration lends itself to model an evolution which is primarily dictated by the migration of the grain boundary. However, with the advancements in material technology, complex polycrystalline systems with chemically-distinct grains are adopted for a wide-range of applications. In these polycrystalline systems with phase- 

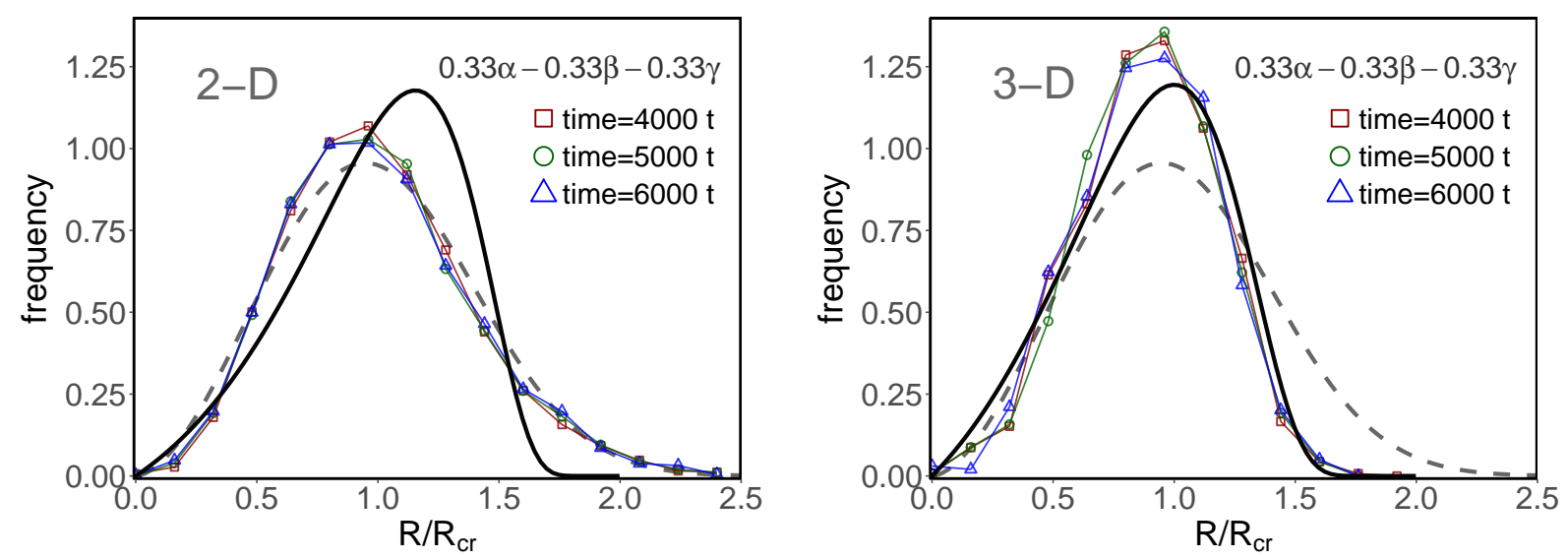

Figure 14: The grain-size distribution of the ternary triplex-microstructure in two- and three-dimension are presented by normalising with critical grain-size. Hillert and Weibull fittings are included to unravel the difference in the grain-size distribution.

associated grains, the energy-minimising transformations like grain growth are no longer exclusively dictated by grain-boundary migration, but is rather convoluted by the phase-fraction constraint. As opposed to the regular grain growth, the corresponding transformation in the multiphase system is accompanied by the temporally unaltered phase-fraction. This characteristic feature renders a large section of the existing techniques inadequate for the investigation of microstructural evolution in multiphase systems. Therefore, in the present works, the ability of a multiphase-field approach to model the concurrent grain growth and coarsening in polycrystalline systems with phase-associated grains is elucidated.

Phase-field techniques have increasingly been used to simulate microstructural transformations. Depending on the treatment of the characteristic scalar variable, the phase-field treatment can be categorised as continuum-field or multiphase-field models. The microstructural evolution in multiphase-field systems have largely been investigated using continuum-field approach. In the current work, the multiphase-field technique is adopted to analyse the temporal evolution polycrystalline systems with phase-associated grains. By comparing different phase-field techniques, a multiphase-field model is derived for a system of multiple chemically-distinct grains, and a provision for incorporating grain boundary and interphase diffusion is discussed.

The effect of number of phases and chemical components on the transformation kinetics is 
investigated by employing multiphase-field approach. It is identified that, while the influence of the phases on the growth rate is direct, the effect of components is governed by the corresponding kinetic coefficients. The increase in the number of phases stunts the evolution by convoluting the diffusion paths followed by the migrating chemical species. In contrast, the increase project 'KerSolife100' and the Helmholtz programme 'Renewable energies' (35.14.01). Authors also thank Ephraim Schoof and Daniel Schneider for the thoughtful discussions. 


\section{Reference}

\section{References}

[1] DG Brandon. The structure of high-angle grain boundaries. Acta metallurgica, 14(11):1479-1484, 1966.

[2] JW Cahn. Stability, microstructural evolution, grain growth, and coarsening in a two-dimensional two-phase microstructure. Acta metallurgica et materialia, 39(10):2189-2199, 1991.

[3] JW Cahn, Elizabeth A Holm, and David J Srolovitz. Modeling microstructural evolution in two-dimensional two-phase microstructures. In Materials Science Forum, volume 94, pages 141-158. Trans Tech Publ, 1992.

[4] DL Jiang, JH Wang, YL Li, and LT Ma. Studies on the strengthening of silicon carbide-based multiphase ceramics I: The SiC TiC system. Materials Science and Engineering: A, 109:401-406, 1989.

[5] Ashkan Keshavarzi, Wolfgang Wisniewski, and Christian Russel. EBSD and EDX analyses of a multiphase glass-ceramic obtained by crystallizing an yttrium aluminosilicate glass. ACS applied materials \& interfaces, 5(17):8531-8536, 2013.

[6] RW Davidge and To J Green. The strength of two-phase ceramic/glass materials. Journal of Materials Science, 3(6):629-634, 1968.

[7] R Filip, K Kubiak, W Ziaja, and J Sieniawski. The effect of microstructure on the mechanical properties of two-phase titanium alloys. Fournal of Materials Processing Technology, 133(1-2):84-89, 2003.

[8] Martin P Harmer, Helen M Chan, and Gary A Miller. Unique opportunities for microstructural engineering with duplex and laminar ceramic composites. Fournal of the American Ceramic Society, 75(7):1715-1728, 1992.

[9] Park Jung Hyun, Hyuk Sang Kwon, et al. Development of high Mn-N duplex stainless steel for automobile structural components. Corrosion Science, 50(2):404-410, 2008.

[10] Park Jung Ihsan-ul Haq Toor and Hyuk Sang Kwon Hyun. Development of high Mn-N duplex stainless steel for automobile structural components. Corrosion Science, 50:404-410, 2008.

[11] EA Holm, David J Srolovitz, and JW Cahn. Microstructural evolution in two-dimensional two-phase polycrystals. Acta metallurgica et materialia, 41(4):1119-1136, 1993.

[12] H Natter, M Schmelzer, M-S Löffler, CE Krill, A Fitch, and R Hempelmann. Grain-growth kinetics of nanocrystalline iron studied in situ by synchrotron real-time X-ray diffraction. The fournal of Physical Chemistry B, 104(11):2467-2476, 2000

[13] Bridget Ingham, Teck H Lim, Christian J Dotzler, Anna Henning, Michael F Toney, and Richard D Tilley. How nanoparticles coalesce: an in situ study of Au nanoparticle aggregation and grain growth. Chemistry of Materials, 23(14):3312-3317, 2011.

[14] BC Larson, Wenge Yang, GE Ice, JD Budai, and JZ Tischler. Three-dimensional X-ray structural microscopy with submicrometre resolution. Nature, 415(6874):887, 2002.

[15] A Gladkikh, Y Lereah, E Glickman, M Karpovski, A Palevski, and J Schubert. Hillock formation during electro- 
migration in $\mathrm{Cu}$ and $\mathrm{Al}$ thin films: Three-dimensional grain growth. Applied physics letters, 66(10):1214-1215, 1995.

[16] Kyozi Kawasaki, Tatsuzo Nagai, and Katsuya Nakashima. Vertex models for two-dimensional grain growth. Philosophical Magazine B, 60(3):399-421, 1989.

[17] LA Barrales Mora, G Gottstein, and LS Shvindlerman. Three-dimensional grain growth: Analytical approaches and computer simulations. Acta Materialia, 56(20):5915-5926, 2008.

[18] Dana Zöllner and Peter Streitenberger. Three-dimensional normal grain growth: Monte Carlo Potts model simulation and analytical mean field theory. Scripta materialia, 54(9):1697-1702, 2006.

[19] PG Kubendran Amos, LT Mushongera, Tobias Mittnacht, and Britta Nestler. Phase-field analysis of volumediffusion controlled shape-instabilities in metallic systems-II: Finite 3-dimensional rods. Computational Materials Science, 144:374-385, 2018.

[20] PG Kubendran Amos, Ephraim Schoof, Daniel Schneider, and Britta Nestler. On the volume-diffusion governed termination-migration assisted globularization in two-phase solid-state systems: Insights from phase-field simulations. In Numerical Modelling in Engineering, pages 47-63. Springer, 2018.

[21] Tobias Mittnacht, PG Kubendran Amos, Daniel Schneider, and Britta Nestler. Understanding the Influence of Neighbours on the Spheroidization of Finite 3-Dimensional Rods in a Lamellar Arrangement: Insights from Phase-Field Simulations. In Numerical Modelling in Engineering, pages 290-299. Springer, 2018.

[22] BI Halperin, PC Hohenberg, and Shang-keng Ma. Renormalization-group methods for critical dynamics: I. Recursion relations and effects of energy conservation. Physical Review B, 10(1):139, 1974.

[23] PG Kubendran Amos, Avisor Bhattacharya, Britta Nestler, and Kumar Ankit. Mechanisms of pearlite spheroidization: Insights from 3D phase-field simulations. Acta Materialia, 161:400-411, 2018.

[24] S-L Wang, RF Sekerka, AA Wheeler, BT Murray, SR Coriell, RJea Braun, and GB McFadden. Thermodynamically-consistent phase-field models for solidification. Physica D: Nonlinear Phenomena, 69(12):189-200, 1993.

[25] Blas Echebarria, Roger Folch, Alain Karma, and Mathis Plapp. Quantitative phase-field model of alloy solidification. Physical Review E, 70(6):061604, 2004.

[26] PG Kubendran Amos, LT Mushongera, and Britta Nestler. Phase-field analysis of volume-diffusion controlled shape-instabilities in metallic systems-I: 2-Dimensional plate-like structures. Computational Materials Science, 144:363-373, 2018.

[27] PG Kubendran Amos, Ephraim Schoof, Nick Streichan, Daniel Schneider, and Britta Nestler. Phase-field analysis of quenching and partitioning in a polycrystalline Fe-C system under constrained-carbon equilibrium condition. Computational Materials Science, 159:281-296, 2019.

[28] Ramanathan Perumal, PG Kubendran Amos, Michael Selzer, and Britta Nestler. Phase-field study on the formation of first-neighbour topological clusters during the isotropic grain growth. Computational Materials Science, 
140:209-223, 2017.

[29] Ramanathan Perumal, PG Kubendran Amos, Michael Selzer, and Britta Nestler. Phase-field study of the transient phenomena induced by 'abnormally'large grains during 2-dimensional isotropic grain growth. Computational Materials Science, 147:227-237, 2018.

[30] PG Kubendran Amos, Ephraim Schoof, Daniel Schneider, and Britta Nestler. On the globularization of the shapes associated with alpha-precipitate of two phase titanium alloys: Insights from phase-field simulations. Acta Materialia, 159:51-64, 2018.

[31] PG Amos. Understanding the volume-diffusion governed shape-instabilities in metallic systems. arXiv preprint arXiv:1906.10404, 2019.

[32] Long-Qing Chen and Danan Fan. Computer Simulation Model for Coupled Grain Growth and Ostwald Ripening-Application to Al2O3-ZrO2 Two-Phase Systems. Journal of the American Ceramic Society, 79(5):1163-1168, 1996.

[33] Stefan Othmar Poulsen, Peter W Voorhees, and Erik Mejdal Lauridsen. Three-dimensional simulations of microstructural evolution in polycrystalline dual-phase materials with constant volume fractions. Acta Materialia, 61(4):1220-1228, 2013.

[34] Hamed Ravash, Jef Vleugels, and Nele Moelans. Three-dimensional phase-field simulation of microstructural evolution in three-phase materials with different diffusivities. Fournal of materials science, 49(20):7066-7072, 2014.

[35] Danan Fan and Long-Qing Chen. Grain growth and microstructural evolution in a two-dimensional two-phase solid containing only quadrijunctions. Scripta materialia, 37(2):233-238, 1997.

[36] Danan Fan and Long-Qing Chen. Topological evolution during coupled grain growth and Ostwald ripening in volume-conserved 2-D two-phase polycrystals. Acta materialia, 45(10):4145-4154, 1997.

[37] Danan Fan and Long-Qing Chen. Diffusion-controlled grain growth in two-phase solids. Acta materialia, 45(8):3297-3310, 1997.

[38] Vishal Yadav, Liesbeth Vanherpe, and Nele Moelans. Effect of volume fractions on microstructure evolution in isotropic volume-conserved two-phase alloys: A phase-field study. Computational Materials Science, 125:297$308,2016$.

[39] Hamed Ravash, Jef Vleugels, and Nele Moelans. Three-dimensional phase-field simulation of microstructural evolution in three-phase materials with different interfacial energies and different diffusivities. Fournal of materials science, 52(24):13852-13867, 2017.

[40] N. Moelans. A quantitative and thermodynamically consistent phase-field interpolation function for multiphase systems. Acta Materialia, 59:1077-1086, 2011.

[41] Long-Qing Chen. Phase-field models for microstructure evolution. Annual review of materials research, 32(1):113-140, 2002. 
[42] Ingo Steinbach. Phase-field models in materials science. Modelling and simulation in materials science and engineering, 17(7):073001, 2009.

[43] Nele Moelans, Frank Wendler, and Britta Nestler. Comparative study of two phase-field models for grain growth. Computational Materials Science, 46(2):479-490, 2009.

[44] Klara Asp and John Agren. Phase-field simulation of sintering and related phenomena-A vacancy diffusion approach. Acta materialia, 54(5):1241-1248, 2006.

[45] Yu U Wang. Computer modeling and simulation of solid-state sintering: A phase field approach. Acta materialia, 54(4):953-961, 2006.

[46] K Ahmed, T Allen, and A El-Azab. Phase field modeling for grain growth in porous solids. Fournal of Materials Science, 51(3):1261-1277, 2016.

[47] Britta Nestler, Harald Garcke, and Björn Stinner. Multicomponent alloy solidification: phase-field modeling and simulations. Physical Review E, 71(4):041609, 2005.

[48] R. Perumal, M. Selzer, and B. Nestler. Concurrent grain growth and coarsening of two-phase microstructures; large scale phase-field study. Computational Materials Science, 159:160-176, 2019.

[49] Ingo Steinbach, Franco Pezzolla, Britta Nestler, Markus Seeßelberg, Robert Prieler, Georg J Schmitz, and Joao LL Rezende. A phase field concept for multiphase systems. Physica D: Nonlinear Phenomena, 94(3):135-147, 1996.

[50] Oleg Tschukin, Alexander Silberzahn, Michael Selzer, Prince GK Amos, Daniel Schneider, and Britta Nestler. Concepts of modeling surface energy anisotropy in phase-field approaches. Geothermal Energy, 5(1):19, 2017.

[51] Seong Gyoon Kim, Won Tae Kim, and Toshio Suzuki. Phase-field model for binary alloys. Physical review e, 60(6):7186, 1999.

[52] J Eiken, B Böttger, and I Steinbach. Multiphase-field approach for multicomponent alloys with extrapolation scheme for numerical application. Physical review E, 73(6):066122, 2006.

[53] VE Badalassi, HD Ceniceros, and Sanjoy Banerjee. Computation of multiphase systems with phase field models. Journal of Computational Physics, 190(2):371-397, 2003.

[54] Mathis Plapp. Unified derivation of phase-field models for alloy solidification from a grand-potential functional. Physical Review E, 84(3):031601, 2011.

[55] Larry K Aagesen, Yipeng Gao, Daniel Schwen, and Karim Ahmed. Grand-potential-based phase-field model for multiple phases, grains, and chemical components. Physical Review E, 98(2):023309, 2018.

[56] Mats Hillert. Phase equilibria, phase diagrams and phase transformations: their thermodynamic basis. Cambridge University Press, 2007.

[57] Nele Moelans, Bart Blanpain, and Patrick Wollants. Quantitative analysis of grain boundary properties in a generalized phase field model for grain growth in anisotropic systems. Physical Review B, 78(2):024113, 2008.

[58] R Folch and M Plapp. Towards a quantitative phase-field model of two-phase solidification. Physical Review E, 68(1):010602, 2003. 
[59] CJ Kuehmann and Peter W Voorhees. Ostwald ripening in ternary alloys. Metallurgical and Materials Transactions A, 27(4):937-943, 1996.

[60] T Philippe and Peter W Voorhees. Ostwald ripening in multicomponent alloys. Acta Materialia, 61(11):42374244, 2013.

[61] LT Mushongera, PG Kubendran Amos, B Nestler, and K Ankit. Phase-field simulations of pearlitic divergence in Fe-C-Mn steels. Acta Materialia, 150:78-87, 2018.

[62] PG Kubendran Amos, Ephraim Schoof, Daniel Schneider, and Britta Nestler. Chemo-elastic phase-field simulation of the cooperative growth of mutually-accommodating Widmanstätten plates. Fournal of Alloys and Compounds, 767:1141-1154, 2018.

[63] Kathleen B Alexander, Paul F Becher, Shirley B Waters, and Alan Bleier. Grain growth kinetics in aluminazirconia (CeZTA) composites. Journal of the American Ceramic Society, 77(4):939-946, 1994.

[64] D Casellas, MM Nagl, L Llanes, and M Anglada. Microstructural Coarsening of Zirconia-Toughened Alumina Composites. Journal of the American Ceramic Society, 88(7):1958-1963, 2005.

[65] HJ Frost, CV Thompson, and DT Walton. Simulation of thin film grain structures-I. Grain growth stagnation. Acta Metallurgica et Materialia, 38(8):1455-1462, 1990.

[66] R Carel, CV Thompson, and HJ Frost. Computer simulation of strain energy effects vs surface and interface energy effects on grain growth in thin films. Acta materialia, 44(6):2479-2494, 1996.

[67] M Hillert. On the theory of normal and abnormal grain growth. Acta metallurgica, 13(3):227-238, 1965.

[68] Chao Wang and Guoquan Liu. On the stability of grain structure with initial Weibull grain size distribution. Materials Letters, 57(28):4424-4428, 2003.

[69] Vishal Yadav and Nele Moelans. Investigation on the existence of a 'Hillert regime'in normal grain growth. Scripta Materialia, 142:148-152, 2018 